\title{
Maternal residential proximity to chlorinated solvent emissions and birth defects in offspring: a case-control study
}

\author{
Jean D Brender ${ }^{1 *}$, Mayura U Shinde ${ }^{1}$, F Benjamin Zhan ${ }^{2}, X_{i}$ Gong $^{2}$ and Peter H Langlois ${ }^{3}$
}

\begin{abstract}
Background: Some studies have noted an association between maternal occupational exposures to chlorinated solvents and birth defects in offspring, but data are lacking on the potential impact of industrial air emissions of these solvents on birth defects.

Methods: With data from the Texas Birth Defects Registry for births occurring in 1996-2008, we examined the relation between maternal residential proximity to industrial air releases of chlorinated solvents and birth defects in offspring of 60,613 case-mothers and 244,927 control-mothers. Maternal residential exposures to solvent emissions were estimated with metrics that took into account residential distances to industrial sources and annual amounts of chemicals released. Logistic regression was used to generate odds ratios and $95 \%$ confidence intervals for the associations between residential proximity to emissions of 14 chlorinated solvents and selected birth defects, including neural tube, oral cleft, limb deficiency, and congenital heart defects. All risk estimates were adjusted for year of delivery and maternal age, education, race/ethnicity, and public health region of residence.

Results: Relative to exposure risk values of 0 , neural tube defects were associated with maternal residential exposures (exposure risk values $>0$ ) to several types of chlorinated solvents, most notably carbon tetrachloride (adjusted odds ratio [aOR] 1.42, 95\% confidence interval [Cl] 1.09, 1.86); chloroform (aOR 1.40, 95\% Cl 1.04, 1.87); ethyl chloride (aOR 1.39, 95\% Cl 1.08, 1.79); 1,1,2-trichloroethane (aOR 1.56, 95\% Cl 1.11, 2.18); and 1,2,3-trichloropropane (aOR 1.49, 95\% Cl 1.08, 2.06). Significant associations were also noted between a few chlorinated solvents and oral cleft, limb deficiency, and congenital heart defects. We observed stronger associations between some emissions and neural tube, oral cleft, and heart defects in offspring of mothers 35 years or older, such as spina bifida with carbon tetrachloride (aOR 2.49, $95 \% \mathrm{Cl} 1.09,5.72)$, cleft palate with 1,2-dichloroethane (aOR 1.93, 95\% 1.05, 3.54), cleft lip with or without cleft palate with ethyl chloride (aOR 1.81,95\% Cl 1.06, 3.07), and obstructive heart defects with trichloroethylene (aOR 1.43, $95 \% \mathrm{Cl} 1.08,1.88)$.
\end{abstract}

Conclusions: These findings suggest that maternal residential proximity to industrial emissions of chlorinated solvents might be associated with selected birth defects in offspring, especially among older mothers.

Keywords: Air pollution, Chlorinated solvents, Congenital heart defects, Limb deficiency defects, Neural tube defects, Oral cleft defects

\footnotetext{
* Correspondence: jdbrender@sph.tamhsc.edu

${ }^{1}$ Texas A\&M Health Science Center School of Public Health, Department of Epidemiology \& Biostatistics, College Station, TX 77843-1266, USA

Full list of author information is available at the end of the article
} 


\section{Background}

Oxidative stress has been suggested as a mechanism by which some teratogens cause birth defects [1], and several chlorinated solvents, such as carbon tetrachloride, chloroform, methylene chloride, and trichloroethylene, have been identified as inducing oxidative stress through giving rise to reactive oxygen species [2]. These compounds have been widely used as solvents for industrial processes, such as metal degreasing and dry cleaning, and for the production of pharmaceuticals, pesticides, adhesives and refrigerants [3]. Findings from several experimental studies of animal models have indicated significantly increased prevalence of various congenital malformations, including exencephaly, musculoskeletal defects and cardiac defects with exposures to some chlorinated solvents [4-8]. These findings were not corroborated, however, by other investigators with experimental studies of rodents exposed to methylene chloride, methyl chloroform, perchloroethylene, and trichloroethylene $[9,10]$.

Several epidemiologic studies have examined the relation between maternal occupational exposure to various chlorinated solvents and birth defects in offspring [11-14] with associations noted between these compounds and cleft lip with or without cleft palate [11], spina bifida [12], limb defects [13], and heart defects [14]. Very limited information has been published on the relation between residential proximity to industrial air releases of chlorinated solvents and birth defects. Yauck et al. [15] examined whether living near industrial facilities in Wisconsin (USA) with trichloroethylene (TCE) emissions increased the risk of congenital heart defects in offspring. Mothers who lived within 1.32 miles of the TCE-emitting facilities had a three-fold excess risk of congenital heart defects, but this association was restricted to mothers aged 38 years and older. Two studies conducted in Texas (USA) $[16,17]$ found no elevated risk of oral cleft or conotruncal heart defects among offspring born to mothers who lived within 1 mile of any industrial facility with reported air emissions of solvents. Increased odds ratios for isolated oral clefts, especially for cleft palate, were noted among offspring to mothers 35 years or older in the Texas study population, although the $95 \%$ confidence intervals for the odds ratios were compatible with the null. In the same study population, residential proximity to industrial facilities with solvent emissions was modestly associated with neural tube defects, and a stronger association was noted in offspring of older mothers [18]. The aforementioned studies of potential maternal ambient air exposures to chlorinated solvents and birth defects did not take into account residential proximity to multiple sites nor the amounts of chemicals released from each of the facilities. Furthermore, the study populations tended to be small, thereby limiting examination of associations of birth defects with specific chemicals by maternal age.
In the present study, we examined whether maternal residential proximity to industrial air emissions of chlorinated solvents was associated with neural tube, oral cleft, limb deficiency, and congenital heart defects in offspring. We accounted for residential proximity to multiple facilities and the annual amounts of specific chlorinated solvents released from each. We also examined whether older maternal age modified associations between residential proximity to chlorinated solvent emissions and birth defects with one of the largest study populations to date.

\section{Methods}

\section{Study population}

Live births, fetal deaths, and induced terminations with selected congenital malformations, including neural tube defects, oral cleft defects, limb deficiencies, and congenital heart defects, were identified from the Texas Birth Defects Registry (TBDR) for births occurring during 1996-2008. The Registry used active surveillance in which trained staff reviewed medical records from hospital, birth centers, and midwifery locations. TBDR covered all cases that were live births, spontaneous fetal deaths (reportable in Texas if 350 grams or more or length of gestation 20 weeks or greater), and pregnancy terminations. Cases were selected in the study if they were delivered between 1996 through 2008, had a maternal address in Texas at time of delivery, and had a diagnosis of one or more of the selected birth defects. The majority of case deliveries were live births (97.1\%) with $1.4 \%$ classified as spontaneous fetal deaths, $1.5 \%$ induced terminations of pregnancy, and $0.1 \%$ unspecified fetal deaths. Live birth and fetal death cases were linked to their respective birth or fetal death certificates that contained information about maternal residential address at the time of delivery and demographic characteristics.

Birth defects were further classified as either isolated or not isolated. Isolated defects were defined as those having a single major defect alone or together with one or more of a list of minor defects used in the National Birth Defects Prevention Study [19].

Control-births, defined as live births without any recorded birth defects, were randomly selected from live birth certificate data obtained from the Center for Health Statistics at the Texas Department of State Health Services. These births were frequency matched to cases by year of delivery (1996-2008) and public health service region (11 regions) in which the case-mothers resided at the time of delivery. We frequency matched birth defect cases and controls by public health region because between 1996 and 1999, active surveillance of birth defect cases did not occur statewide, but was confined to certain regions, including 2 regions in 1996, 6 regions in 1997, and 8 regions in 1998. These regions range from 12,060 (Region 5) to 61,456 square miles (Regions $9 / 10$ combined). 
ESRI ArcGIS 8.3 ArcMap 9.3 was used to geocode the maternal addresses obtained from live birth and fetal death certificates. A total of $86.7 \%$ of the maternal addresses for case-mothers and $87.2 \%$ for those of controlmothers were successfully geocoded to street level.

Linkage to industrial air emissions of chlorinated solvents Data regarding air emissions from Texas industrial facilities were obtained from the U.S. Environmental Protection Agency Toxic Release Inventory (TRI) program. The online TRI databases contain names of facilities required to report under section 313 of the Emergency Planning and Community Right-to-Know Act and include information about location, reporting year, chemicals released, and estimated pounds per year released into various environmental media, such as air and water bodies. For the present project, industrial air releases of 14 chlorinated solvents were identified for study, including carbon tetrachloride; chloroform; 1,1-dichloroethane; 1,2-dichloroethane; 1,2-dichloroethylene; ethyl chloride; methyl chloroform; methylene chloride; perchloroethylene; propylene dichloride; tetrachloroethane; 1,1,2-trichloroethane, trichloroethylene; and 1,2,3-trichloropropane.

Based on the addresses available in the USEPA TRI databases, we geocoded the TRI facilities in three steps. The addresses were first geocoded using Centrus Geocoder for ArcGIS. Then, the TRI addresses that were not geocoded in the first step were geocoded by the default geocoding tools from ArcGIS 10.0 using the Census 2000 street map as the reference layer. Finally, the remaining TRI addresses that were not successfully geocoded using the first two methods were geocoded with Google map geocoding API. Only TRI addresses geocoded to the premise level were considered successful matches. Over the 13 year period of 1996-2008, approximately 90\% of the industrial facilities, on average, were successfully geocoded.

To estimate potential intensities of various chlorinated solvent air releases in the vicinity of maternal residences, a modified version of the Emission Weighted Proximity Model (EWPM) was used [20]. This model takes into consideration emissions from all sources within a $10-\mathrm{km}$ effective threshold potentially affecting a person at a specific location and the amounts of chemical released from each source during a given time period [20]. The formula for calculating the combined quantity of air pollu$\operatorname{tant} \theta$ at location $i$ from all emission sources is given by Expression (1).

$$
A^{\theta}{ }_{i}=\sum_{j=1}^{m} E^{\theta}{ }_{i j} \times T^{\theta}{ }_{i j} \times\left(\left(\left(k^{\theta}-D_{i j}\right) / k^{\theta}\right), \text { for }\left(D_{i j} \leq k^{\theta}\right)\right.
$$

where $A^{\theta}{ }_{i}$ is the estimated quantity of air pollutant $\theta$ at location $i$ from all emission sources $j(j=1,2, \ldots . ., m)$ within the threshold distance. This quantity was used as a proxy to represent the exposure risk value of a person at location $i$ to air pollutant $\theta ; m$ is the number of emission sources relevant to a person at location $i$ in the area in question; $E^{\theta}{ }_{i j}$ is the emission rate of air pollutant $\theta$ from any emission source $j$ that is within the effective threshold distance related to location $i ; T^{\theta}{ }_{i j}$ is the duration of emission of air pollutant $\theta$ from emission source $j ; k^{\theta}$ is effective threshold distance beyond which air pollutant $\theta$ is considered to have no harm to an individual and is determined by the physical and photochemistry characteristics of the pollutant in question [19]; and $D_{i j}$ is the distance between location $i$ and location of $j$. Based on Expression (1), an exposure risk value for each of the 14 chlorinated solvents was assigned to case- and control-mothers based on their residence location at the time of delivery. The TRI facilities and maternal addresses were linked through the EWPM. Given the effective threshold distance, only the facilities within the distance of a maternal address were considered to have potential harm on that person.

\section{Data analyses}

The EWPM exposure risk values were categorized into two groups (exposure risk values for a given solvent equaling zero or greater than zero) and four or seven levels (exposure risk values at zero and greater than zero divided into three or six equal groups) based on the control-mothers distribution of scores. In the analyses with more than two categories of exposure risk values, the number of categories used (4 or 7 ) depended on the numbers of cases available. We used logistic regression to examine the relation (odds ratio [OR] and 95\% confidence interval $[\mathrm{CI}]$ ) between maternal residential exposures to chlorinated solvents and neural tube defects, oral clefts, limb deficiencies, and selected heart defects in offspring. The lowest level of exposure risk value served as the referent group for all analyses. In the analyses of odds ratios associated with varying intensities of exposure risk values, the Wald statistic was used to test for significance of linear trends. To reduce potential confounding, the ORs were adjusted for the year of delivery (1996-2008), maternal age $(<20,20-24,25-29,30-34,35-39,>39$ years $)$, education ( $<12$ years, 12 years, $>12$ years), race/ethnicity (white non-Hispanic, black non-Hispanic, Hispanic, other non-Hispanic), and public health region of residence (11 regions in Texas).

Because sufficient numbers of exposed cases were available, analyses of the association between chlorinated solvents and oral clefts were restricted to isolated defects. Analyses of the other types of birth defects included all identified cases. Heart defects were grouped into five major categories including conotruncal defects (truncus arteriosus, transposition of the great arteries, 
double outlet right ventricle, and Tetralogy of Fallot); obstructive heart defects (Ebstein anomaly, aortic valve stenosis, hypoplastic left heart syndrome, coarctation of the aorta, pulmonary artery atresia); septal defects (ventricular septal defect, atrial septal defect); atrioventricular septal defects (atrial septal defect primum, single common atrium, complete atrioventricular canal, endocardial cushion defect); and anomalous pulmonary venous return.

We also examined the effect of maternal age on the risk of selected birth defects associated with higher residential exposures to solvents. The EWPM exposure risk values as dichotomous variables $(0,>0)$ were stratified by categories of maternal age ( $<35$ years and 35 years or older) and agespecific ORs and respective 95\% CIs were calculated. Additive and multiplicative interactions were assessed for the associations of birth defects with EWPM exposure risk values that appeared to vary by maternal age. Additive interaction was examined using a statistical program developed by Andersson et al. that estimated measures of relative excess risk due to interaction (RERI) and attributable proportion due to interaction (AP) [21]. If either or both measures differed from zero and their $95 \%$ CIs excluded 0 , significant additive interaction was considered present. To assess multiplicative interaction, the product terms of EWPM risk values for a given solvent with maternal age were included in the logistic models and were considered significant if the p-value was less than 0.05 .

\section{Results}

Table 1 provides a comparison of case-mothers (of deliveries with neural tube defects, limb deficiencies, oral clefts, and congenital heart defects) and control mothers by demographic characteristics, delivery year, public health region of residence at the time of delivery, and geocoding status of residence. A total of 3245 cases with neural tube defects, 2406 cases with limb deficiencies, 7416 cases with oral clefts, 60154 cases with selected heart defects, and 280764 controls without major birth defects were available for the study. Compared with control-mothers, mothers of neural tube defect cases were more likely to be Hispanic and have less education, while mothers of babies with limb deficiencies and oral clefts were more likely to be non-Hispanic White. Mothers with babies with limb deficiencies and oral clefts were more likely than controlmothers to smoke during pregnancy, although smoking appeared underreported overall, given the low prevalence reported on the birth and fetal death certificates. Maternal residential street addresses were successfully geocoded to street level for $87.2 \%, 69.1 \%, 83.9 \%, 84.7 \%$, and $87.4 \%$ respectively for control-births, and births with neural tube defects, limb deficiencies, oral cleft defects, and heart defects. Maternal addresses for neural tube defect cases were less likely to be geocoded because $21 \%$ of these deliveries were induced terminations for which maternal residential addresses were unavailable.

Neural tube defects were significantly associated with maternal residential proximity to several types of chlorinated solvent air emissions (Table 2), most notably with carbon tetrachloride (adjusted odds ratio [aOR] 1.42, 95\% CI 1.09, 1.86); chloroform (aOR 1.40, 95\% CI 1.04, 1.87); 1,2 dichloroethane (aOR 1.28, 95\% CI 1.01, 1.62); ethyl chloride (aOR 1.39, 95\% CI 1.08, 1.79); methyl chloroform (aOR 1.29, 95\% CI 1.01, 1.63); 1,1,2 trichloroethane (aOR $1.56,95 \%$ CI 1.11, 1.28); and 1,2,3 trichloropropane (aOR $1.49,95 \%$ CI 1.08, 2.06). Further examination of the neural tube defect phenotypes revealed that spina bifida, but not anencephaly, was associated with these chemicals. Spina bifida was also associated with maternal residential proximity to emissions of 1,1-dichloroethane (aOR 1.70, 95\% CI 1.06, 2.71), 1,2 dichloroethylene (aOR 1.60, 95\% CI 1.01, 2.53), and tetrachloroethane (aOR 1.78, 95\% CI 1.12, 2.82).

With respect to chlorinated solvent emissions and oral clefts, a few positive associations were observed with cleft palate alone (Table 3). Propylene dichloride, in particular, was associated with cleft palate (aOR 1.77, 95\% CI 1.05, 2.99).

In analyses of congenital heart defects, only septal heart defects showed a significant association with emissions of chlorinated solvents, and these associations tended to be weak with the ORs ranging from 1.06 to 1.23 (Table 4, data for atrioventricular heart defects and anomalous venous return not shown). The only associations noted with limb defects included perchloroethylene with transverse limb deficiencies (aOR 1.21, $95 \%$ CI 1.01, 1.45) (Table 5) and any type of chlorinated solvent (exposure risk values summed for 14 chemicals) with lower limb deficiencies (aOR 1.21, 95\% CI 1.00, 1.45 , data not shown).

Tables 6,7 , and 8 display results of analyses of chlorinated solvents with spina bifida, cleft palate alone, and septal heart defects, respectively, in which exposure risk values were divided into four groups (zero, low, middle, high) based on the control-mother's distributions. Significant linear trends were noted between the emissions of several chlorinated solvents and spina bifida, including chloroform; 1,1-dichloroethane; 1,2-dichloroethane; ethyl chloride; methyl chloride; tetrachloroethane; 1,1,2-trichloroethane; and 1,2,3-trichloropropane (Table 6). The strongest linear trends were noted with 1,2-dichloroethane (aOR for spina bifida in the fourth quartile: 1.85, 95\% CI $1.21,2.84$ ) and with 1,2,3-trichloropropane (aOR in the fourth quartile: $2.62,95 \%$ CI 1.55, 4.46). Several of the trends were not monotonic in that the highest odds ratios were noted in the second quartile versus the fourth quartile.

Although the trends were not uniformly monotonic, the strongest associations between cleft palate alone and 
Table 1 Selected maternal characteristics of birth defect cases and controls, Texas Birth Defects Registry, 1996-2008

\begin{tabular}{|c|c|c|c|c|c|c|c|c|c|c|}
\hline \multirow[t]{2}{*}{ Characteristic } & \multicolumn{2}{|c|}{$\begin{array}{c}\text { Controls } \\
(\mathrm{n}=\mathbf{2 8 0 7 6 4 )}\end{array}$} & \multicolumn{2}{|c|}{$\begin{array}{c}\text { Neural tube } \\
\text { defects }(n=3245)\end{array}$} & \multicolumn{2}{|c|}{$\begin{array}{c}\text { Limbs deficiencies } \\
(n=2406)\end{array}$} & \multicolumn{2}{|c|}{$\begin{array}{l}\text { Oral cleft defects } \\
(n=7416)\end{array}$} & \multicolumn{2}{|c|}{$\begin{array}{l}\text { Heart defects } \\
(n=60154)\end{array}$} \\
\hline & $n$ & $\%$ & $n$ & $\%$ & $n$ & $\%$ & $n$ & $\%$ & $n$ & $\%$ \\
\hline \multicolumn{11}{|l|}{ Race-ethnicity } \\
\hline Non-Hispanic white & 93425 & 33.3 & 956 & 31.7 & 890 & 38.3 & 2831 & 39.0 & 20434 & 34.2 \\
\hline Non-Hispanic black & 27119 & 9.7 & 246 & 8.1 & 257 & 11.1 & 542 & 7.5 & 6144 & 10.3 \\
\hline Hispanic & 150334 & 53.6 & 1749 & 58.0 & 1114 & 48.0 & 3617 & 49.8 & 31441 & 52.7 \\
\hline Others, non-Hispanic & 9510 & 3.4 & 66 & 2.2 & 61 & 2.6 & 277 & 3.8 & 1670 & 2.8 \\
\hline Missing & 376 & & 228 & & 84 & & 149 & & 465 & \\
\hline \multicolumn{11}{|l|}{ Education } \\
\hline$<$ High school & 91641 & 32.9 & 998 & 39.2 & 732 & 32.9 & 2424 & 34.2 & 19523 & 33.1 \\
\hline High school & 82138 & 29.5 & 785 & 30.9 & 691 & 31.0 & 2176 & 30.7 & 17448 & 29.5 \\
\hline >High school & 104427 & 37.5 & 760 & 29.9 & 804 & 36.1 & 2481 & 35.0 & 22059 & 37.4 \\
\hline Missing & 2558 & & 702 & & 179 & & 335 & & 1124 & \\
\hline \multicolumn{11}{|l|}{ Age at delivery (years) } \\
\hline $11-19$ & 41376 & 14.7 & 462 & 15.2 & 379 & 16.3 & 1063 & 14.6 & 7973 & 13.3 \\
\hline $20-24$ & 79641 & 28.4 & 860 & 28.3 & 647 & 27.8 & 2014 & 27.7 & 15433 & 25.8 \\
\hline $25-29$ & 74994 & 26.7 & 823 & 27.1 & 609 & 26.2 & 1915 & 26.3 & 15145 & 25.3 \\
\hline $30-34$ & 55326 & 19.7 & 579 & 19.0 & 438 & 18.8 & 1378 & 18.9 & 12050 & 20.2 \\
\hline $35-39$ & 24487 & 8.7 & 248 & 8.2 & 184 & 7.9 & 689 & 9.5 & 6977 & 11.7 \\
\hline$>39$ & 4912 & 1.8 & 68 & 2.2 & 68 & 2.9 & 216 & 3.0 & 2193 & 3.7 \\
\hline Missing & 28 & & 205 & & 81 & & 141 & & 383 & \\
\hline \multicolumn{11}{|c|}{ Public health service region } \\
\hline 1 & 9368 & 3.3 & 93 & 3.0 & 91 & 3.9 & 296 & 4.1 & 1957 & 3.3 \\
\hline 2 & 5864 & 2.1 & 70 & 2.3 & 44 & 1.9 & 199 & 2.7 & 1219 & 2.0 \\
\hline 3 & 76992 & 27.4 & 921 & 30.2 & 730 & 31.4 & 2092 & 28.7 & 16117 & 27.0 \\
\hline 4 & 7080 & 2.5 & 106 & 3.5 & 82 & 3.5 & 285 & 3.9 & 1351 & 2.3 \\
\hline 5 & 4640 & 1.7 & 48 & 1.6 & 48 & 2.1 & 161 & 2.2 & 950 & 1.6 \\
\hline 6 & 50124 & 17.9 & 532 & 17.5 & 390 & 16.8 & 1395 & 19.2 & 10557 & 17.7 \\
\hline 7 & 22544 & 8.0 & 259 & 8.5 & 230 & 9.9 & 723 & 9.9 & 4594 & 7.7 \\
\hline 8 & 28740 & 10.2 & 314 & 10.3 & 296 & 12.7 & 778 & 10.7 & 6053 & 10.1 \\
\hline 9 & 6932 & 2.5 & 84 & 2.8 & 60 & 2.6 & 202 & 2.8 & 1441 & 2.4 \\
\hline 10 & 8576 & 3.1 & 131 & 4.3 & 84 & 3.6 & 309 & 4.3 & 1702 & 2.8 \\
\hline 11 & 59904 & 21.3 & 487 & 16.0 & 272 & 11.7 & 839 & 11.5 & 13838 & 23.1 \\
\hline Missing & - & & 200 & & 79 & & 137 & & 375 & \\
\hline \multicolumn{11}{|l|}{ Year of birth } \\
\hline 1996 & 6964 & 2.5 & 125 & 4.1 & 61 & 2.6 & 181 & 2.5 & 1444 & 2.4 \\
\hline 1997 & 12648 & 4.5 & 170 & 5.6 & 102 & 4.4 & 355 & 4.9 & 2659 & 4.5 \\
\hline 1998 & 16468 & 5.9 & 222 & 7.3 & 153 & 6.6 & 476 & 6.5 & 3398 & 5.7 \\
\hline 1999 & 19096 & 6.8 & 287 & 9.4 & 186 & 8.0 & 575 & 7.9 & 3866 & 6.5 \\
\hline 2000 & 20040 & 7.1 & 257 & 8.4 & 186 & 8.0 & 616 & 8.5 & 4092 & 6.9 \\
\hline 2001 & 20588 & 7.3 & 232 & 7.6 & 204 & 8.8 & 605 & 8.3 & 4243 & 7.1 \\
\hline 2002 & 21580 & 7.7 & 226 & 7.4 & 192 & 8.3 & 574 & 7.9 & 4566 & 7.6 \\
\hline 2003 & 22980 & 8.2 & 232 & 7.6 & 192 & 8.3 & 579 & 8.0 & 4927 & 8.2 \\
\hline 2004 & 25428 & 9.1 & 264 & 8.7 & 183 & 7.9 & 646 & 8.9 & 5468 & 9.2 \\
\hline
\end{tabular}


Table 1 Selected maternal characteristics of birth defect cases and controls, Texas Birth Defects Registry, 1996-2008 (Continued)

\begin{tabular}{|c|c|c|c|c|c|c|c|c|c|c|}
\hline 2005 & 27464 & 9.8 & 248 & 8.1 & 211 & 9.1 & 672 & 9.2 & 5981 & 10.0 \\
\hline 2006 & 27548 & 9.8 & 248 & 8.1 & 213 & 9.2 & 648 & 8.9 & 6004 & 10.0 \\
\hline 2007 & 28828 & 10.3 & 266 & 8.7 & 228 & 9.8 & 665 & 9.1 & 6286 & 10.5 \\
\hline 2008 & 31132 & 11.1 & 269 & 8.8 & 216 & 9.3 & 688 & 9.4 & 6846 & 11.4 \\
\hline Missing & - & - & 199 & & 79 & & 136 & & 374 & \\
\hline \multicolumn{11}{|l|}{ Smoking ${ }^{a}$} \\
\hline No & 230492 & 94.5 & 2061 & 94.9 & 1802 & 92.5 & 5684 & 92.2 & 48978 & 94.1 \\
\hline Yes & 13338 & 5.5 & 111 & 5.1 & 145 & 7.5 & 479 & 7.8 & 3061 & 5.9 \\
\hline Missing & 36934 & & 1073 & & 459 & & 1253 & & 8115 & \\
\hline \multicolumn{11}{|l|}{ Geocode accuracy } \\
\hline 9-Digit Zip & 684 & 0.2 & 4 & 0.1 & 3 & 0.1 & 18 & 0.2 & 135 & 0.2 \\
\hline Manual & 299 & 0.1 & 19 & 0.6 & 4 & 0.2 & 17 & 0.2 & 55 & 0.1 \\
\hline Street & 244927 & 87.2 & 2241 & 69.1 & 2018 & 83.9 & 6282 & 84.7 & 52591 & 87.4 \\
\hline Zip & 5955 & 2.1 & 42 & 1.3 & 39 & 1.6 & 156 & 2.1 & 1133 & 1.9 \\
\hline Not Geocoded & 28899 & 10.3 & 267 & 8.2 & 197 & 8.2 & 686 & 9.3 & 5737 & 9.5 \\
\hline Missing & - & - & 672 & 20.7 & 145 & 6.0 & 257 & 3.5 & 503 & 0.8 \\
\hline
\end{tabular}

${ }^{a}$ Anytime during pregnancy.

Table 2 Maternal residential proximity to air emissions of chlorinated solvents and neural tube defects, Texas, 1996-2008

\begin{tabular}{|c|c|c|c|c|c|c|c|c|c|c|c|c|c|c|}
\hline \multirow{3}{*}{$\begin{array}{c}\text { Type of } \\
\text { chlorinated solvent }\end{array}$} & \multicolumn{14}{|c|}{ Exposure risk value $>0^{a}$} \\
\hline & \multicolumn{2}{|c|}{ Controls } & \multicolumn{2}{|c|}{$\begin{array}{l}\text { Neural tube } \\
\text { defect cases }\end{array}$} & \multicolumn{2}{|c|}{$\begin{array}{l}\text { Adjusted odds } \\
\text { ratio }^{\mathbf{b}}(95 \% \mathrm{Cl})\end{array}$} & \multicolumn{2}{|c|}{$\begin{array}{l}\text { Anencephaly } \\
\text { cases }\end{array}$} & \multicolumn{2}{|c|}{$\begin{array}{l}\text { Adjusted odds } \\
\text { ratio }^{\mathbf{b}}(95 \% \mathrm{Cl})\end{array}$} & \multicolumn{2}{|c|}{$\begin{array}{l}\text { Spina bifida } \\
\text { cases }\end{array}$} & \multicolumn{2}{|c|}{ 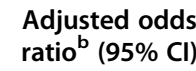 } \\
\hline & $n$ & $\%$ & $n$ & $\%$ & & & $n$ & $\%$ & & & $n$ & $\%$ & & \\
\hline Any type & 63599 & 26.2 & 619 & 28.4 & 0.98 & $(0.88,1.09)$ & 173 & 29.3 & 1.04 & $(0.84,1.27)$ & 371 & 28.3 & 0.97 & $(0.85,1.11)$ \\
\hline loride & 4568 & 1.9 & 6 & 2.8 & 1.42 & 86) & 13 & 2.2 & 1.18 & $(0.66$ & 44 & 3.4 & 1.58 & 2.19 \\
\hline Chloroform & 3985 & 1.6 & 52 & 2.4 & 1.40 & $(1.04,1.87)$ & 10 & 1.7 & 1.09 & $(0.57,2.09)$ & 37 & 2.8 & 1.55 & $(1.10,2.20$ \\
\hline 1-dichloroethane & 1847 & 0.8 & 24 & 1.1 & 1.36 & $(0.90,2.05)$ & 5 & 0.8 & 1.13 & $(0.46,2.77)$ & 19 & 1.4 & 1.70 & $(1.06,2.7$ \\
\hline 1,2-dichloroethane & 6637 & 2.7 & 85 & 3.9 & 1.28 & $(1.01,1.62)$ & 13 & 2.2 & 0.69 & $(0.39,1.23)$ & 65 & 5.0 & 1.64 & $(1.24,2.1$ \\
\hline 2-dichlo & 2026 & 0.8 & 25 & 1.1 & 1.26 & ) & 5 & 0.8 & 0.99 & $(C$ & 20 & 1.5 & 1.60 & $(1.01,2.53$ \\
\hline Ethyl chloride & 5271 & 2.2 & 70 & 3.2 & 1.39 & $(1.08,1.79)$ & 16 & 2.7 & 1.28 & $(0.76,2.15)$ & 50 & 3.8 & 1.59 & $(1.18,2.14$ \\
\hline Methyl chloroform & 5294 & 2.2 & 79 & 3.6 & 1.29 & $(1.01,1.63)$ & 15 & 2.5 & 0.85 & $(0.50,1.44)$ & 57 & 4.3 & 1.56 & $(1.18,2.0$ \\
\hline Methylene chloride & 38448 & 15.9 & 401 & 18.4 & 1.03 & $(0.91,1.16)$ & 109 & 18.4 & 1.03 & $(0.82,1.30)$ & 240 & 18.3 & 1.02 & $(0.87,1.18)$ \\
\hline erchloroethylene & 29854 & 12.3 & 277 & 12.7 & 0.92 & $(0.81,1.06)$ & 78 & 13.2 & 0.95 & $(0.73,1.22)$ & 162 & 12.3 & 0.90 & $(0.75,1.0$ \\
\hline Propylene dichloride & 1518 & 0.6 & 16 & 0.7 & 1.15 & $(0.70,1.90)$ & 1 & 0.2 & $-^{c}$ & - & 14 & 1.1 & 1.58 & $(0.92,2.7$ \\
\hline Tetrachloroethane & 1955 & 0.8 & 23 & 1.1 & 1.31 & $(0.86,2.00)$ & 3 & 0.5 & $-^{c}$ & - & 20 & 1.5 & 1.78 & $(1.12,2.82)$ \\
\hline 1,1,2-trichloroethane & 2561 & 1.1 & 38 & 1.7 & 1.56 & $(1.11,2.18)$ & 0 & 1.0 & 0.97 & $(0.42,2.21)$ & 30 & 2.3 & 1.94 & $(1.32,2.8$ \\
\hline Trichloroethylene & 28547 & 11.8 & 274 & 12.6 & 0.95 & $(0.83,1.09)$ & 78 & 13.2 & 0.99 & $(0.76,1.29)$ & 163 & 12.4 & 0.94 & $(0.79,1.12$ \\
\hline 1,2,3-trichloropropane & 2847 & 1.2 & 41 & 1.9 & 1.49 & $(1.08,2.06)$ & 8 & 1.4 & 1.15 & $(0.56,2.36)$ & 31 & 2.4 & 1.78 & $(1.22,2.5$ \\
\hline
\end{tabular}

${ }^{a}$ Exposure risk value based on residential proximity to source(s) of air emissions and estimated pounds of chemical emitted annually.

${ }^{\mathrm{b}}$ Adjusted for birth year and maternal age, education, race/ethnicity, and public health region of residence (referent group: exposure risk value $=0$ ).

'Odds ratios and respective $95 \%$ confidence intervals are not reported for analyses with less than five exposed cases. 
Table 3 Maternal residential proximity to air emissions of chlorinated solvents and isolated oral cleft defects, Texas, 1996-2008

\begin{tabular}{|c|c|c|c|c|c|c|c|c|c|c|c|c|c|c|}
\hline \multirow{3}{*}{$\begin{array}{c}\text { Type of } \\
\text { chlorinated solvent }\end{array}$} & \multicolumn{14}{|c|}{ Exposure risk value $>0^{a}$} \\
\hline & \multicolumn{2}{|c|}{ Controls } & \multicolumn{2}{|c|}{$\begin{array}{l}\text { Any oral cleft } \\
\text { defect }\end{array}$} & \multicolumn{2}{|c|}{$\begin{array}{l}\text { Adjusted odds } \\
\text { ratio }^{\mathrm{b}}(95 \% \mathrm{Cl})\end{array}$} & \multicolumn{2}{|c|}{$\begin{array}{l}\text { Cleft palate } \\
\text { alone }\end{array}$} & \multicolumn{2}{|c|}{$\begin{array}{l}\text { Adjusted odds } \\
\text { ratio }^{\mathrm{b}}(95 \% \mathrm{Cl})\end{array}$} & \multicolumn{2}{|c|}{$\begin{array}{l}\text { Cleft lip with or } \\
\text { without cleft palate }\end{array}$} & \multicolumn{2}{|c|}{$\begin{array}{l}\text { Adjusted odds } \\
\text { ratio b }^{\text {b }}(95 \% \mathrm{Cl})\end{array}$} \\
\hline & $n$ & $\%$ & $n$ & $\%$ & & & $n$ & $\%$ & & & $n$ & $\%$ & & \\
\hline Any type & 63599 & 26.2 & 1036 & 26.8 & 0.99 & $(0.91,1.07)$ & 308 & 26.5 & 0.95 & $(0.82,1.10)$ & 729 & 27.0 & 1.00 & $(0.91,1.10)$ \\
\hline Carbon tetrachloride & 4568 & 1.9 & 91 & 2.4 & 1.10 & $(0.88,1.37)$ & 32 & 2.8 & 1.25 & $(0.86,1.80)$ & 59 & 2.2 & 1.03 & $(0.79,1.36)$ \\
\hline Chloroform & 3985 & 1.6 & 79 & 2.0 & 1.05 & $(0.83,1.33)$ & 25 & 2.1 & 1.03 & $(0.68,1.56)$ & 54 & 2.0 & 1.06 & $(0.80,1.40)$ \\
\hline 1,1-dichloroethane & 1847 & 0.8 & 33 & 0.9 & 1.00 & $(0.70,1.42)$ & 13 & 1.1 & 1.24 & $(0.71,2.16)$ & 20 & 0.7 & 0.89 & $(0.57$ \\
\hline 1,2-dichloroethane & 6637 & 2.7 & 119 & 3.1 & 0.99 & $(0.81,1.20)$ & 45 & 4.1 & 1.10 & $(0.79,1.54)$ & 78 & 2.9 & 0.94 & $(0.74,1.2$ \\
\hline 1,2-dichloroethylene & 2026 & 0.8 & 38 & 1.0 & 1.04 & $(0.75,1.44)$ & 14 & 1.2 & 1.19 & $(0.69,2.04)$ & 24 & 0.9 & 0.97 & $(0.64,1.4)$ \\
\hline Ethyl chloride & 5271 & 2.2 & 107 & 2.8 & 1.10 & $(0.90,1.34)$ & 34 & 2.9 & 1.09 & $(0.77,1.56)$ & 73 & 2.7 & 1.11 & $(0.87,1.41$ \\
\hline Methyl chloroform & 5294 & 2.2 & 105 & 2.7 & 1.06 & $(0.87,1.30)$ & 30 & 2.6 & 0.99 & $(0.68,1.45)$ & 75 & 2.8 & 1.09 & $(0.86,1.3$ \\
\hline Methylene chloride & 38448 & 15.9 & 672 & 17.4 & 1.06 & $(0.97,1.16)$ & 193 & 16.6 & 0.99 & $(0.84,1.17)$ & 480 & 17.8 & 1.09 & $(0.98,1.21)$ \\
\hline Perchloroethylene & 29854 & 12.3 & 462 & 12.0 & 0.98 & $(0.88,1.08)$ & 127 & 10.9 & 0.88 & $(0.72,1.07)$ & 335 & 12.4 & 1.02 & $(0.90,1.1$ \\
\hline Propylene dichloride & 1518 & 0.6 & 29 & 0.8 & 1.07 & $(0.74,1.56)$ & 15 & 1.3 & 1.77 & $(1.05,2.99)$ & 14 & 0.5 & 0.75 & $(0.44,1.28$ \\
\hline Tetrachloroethane & 1955 & 0.8 & 36 & 0.9 & 1.05 & $(0.75,1.48)$ & 17 & 1.5 & 1.61 & $(0.98,2.64)$ & 19 & 0.7 & 0.81 & $(0.51,1.28$ \\
\hline 1,1,2-trichloroethane & 2561 & 1.1 & 54 & 1.4 & 1.16 & $(0.87,1.53)$ & 22 & 1.9 & 1.51 & $(0.97,2.34)$ & 32 & 1.2 & 1.00 & $(0.70,1.4$ \\
\hline Trichloroethylene & 28547 & 11.8 & 483 & 12.5 & 1.02 & $(0.92,1.13)$ & 144 & 12.4 & 1.00 & $(0.83,1.21)$ & 339 & 12.5 & 1.02 & $(0.90,1.16)$ \\
\hline 1,2,3-trichloropropane & 2847 & 1.2 & 61 & 1.6 & 1.16 & $(0.89,1.51)$ & 24 & 2.1 & 1.48 & $(0.97,2.25)$ & 37 & 1.4 & 1.03 & $(0.73,1.4$ \\
\hline
\end{tabular}

${ }^{a}$ Exposure risk value based on residential proximity to source(s) of air emissions and estimated pounds of chemical emitted annually.

${ }^{\mathrm{b}}$ Adjusted for birth year and maternal age, education, race/ethnicity, and public health region of residence (referent group: exposure risk value $=0$ ).

Table 4 Maternal residential proximity to air emissions of chlorinated solvents and congenital heart defects, Texas, 1996-2008

\begin{tabular}{|c|c|c|c|c|c|c|c|c|c|c|c|c|c|c|}
\hline \multirow{3}{*}{$\begin{array}{c}\text { Type of } \\
\text { chlorinated solvent }\end{array}$} & \multicolumn{14}{|c|}{ Exposure risk value $>0^{a}$} \\
\hline & \multicolumn{2}{|c|}{ Controls } & \multicolumn{2}{|c|}{$\begin{array}{l}\text { Conotruncal } \\
\text { heart defects }\end{array}$} & \multicolumn{2}{|c|}{$\begin{array}{l}\text { Adjusted odds } \\
\text { ratio }^{\mathrm{b}}(95 \% \mathrm{Cl})\end{array}$} & \multicolumn{2}{|c|}{$\begin{array}{c}\text { Obstructive } \\
\text { heart defects }\end{array}$} & \multicolumn{2}{|c|}{$\begin{array}{l}\text { Adjusted odds } \\
\text { ratio }^{\mathrm{b}}(95 \% \mathrm{Cl})\end{array}$} & \multicolumn{2}{|c|}{$\begin{array}{l}\text { Septal heart } \\
\text { defects }\end{array}$} & \multicolumn{2}{|c|}{$\begin{array}{l}\text { Adjusted odds } \\
\text { ratio }^{\text {b }}(95 \% \mathrm{Cl})\end{array}$} \\
\hline & $n$ & $\%$ & $n$ & $\%$ & & & $n$ & $\%$ & & & $n$ & $\%$ & & \\
\hline ny type & 63599 & 26.2 & 941 & 28.5 & 1.05 & $(0.96,1.14)$ & 912 & 26.9 & 1.02 & $(0.93,1.11)$ & 11325 & 26.4 & 1.06 & $(1.04,1.09)$ \\
\hline arbon tetrachloride & 4568 & 1.9 & ' & 2.3 & 1.09 & $(0.86,1.39)$ & 02 & 2.4 & 1.16 & $(0.92$, & 867 & 2.0 & 1.13 & 1.22 \\
\hline Chloroform & 3985 & 1.6 & 60 & 1.8 & 0.97 & $(0.74$ & 0 & 2.1 & 1.13 & $(0.88$ & 739 & 1.7 & 1.10 & $(1.01,1.1$ \\
\hline 1,1-dichloroethane & 1847 & 0.8 & 3 & 0.9 & 1. & $(C$ & 34 & 1.0 & 1.17 & $(C$ & 387 & 0.9 & 1.23 & $(1.10,1.3)$ \\
\hline 1,2-dichloroethane & 6637 & 2.7 & 102 & 3.1 & 1.00 & $(0.81,1.24)$ & 106 & 3.1 & 1.03 & $(0.83,1.26)$ & 1184 & 2.8 & 1.06 & $(0.99,1.1$ \\
\hline 1,2-dichloroe & 2026 & 0.8 & 3 & 1.0 & 1.13 & $(0.80$ & 8 & 1.1 & 1.20 & $(0.86$ & 409 & 1.0 & 1.19 & (10 \\
\hline Ethyl chloride & 5271 & 2.2 & 82 & 2.5 & 1.02 & $(0.81,1.28)$ & 98 & 2.9 & 1.23 & $(0.99,1.51)$ & 1002 & 2.3 & 1.13 & $(1.05,1.2$ \\
\hline lethyl chlor & 5294 & 2.2 & 78 & 2.4 & 0.97 & $(0.77,1.23)$ & 03 & 2.7 & 1.14 & $(0.92,1$ & 865 & 2.0 & 1.01 & $(0.94,1.0$ \\
\hline Methylene chloride & 38448 & 15.9 & 583 & 17.6 & 1.04 & $(0.95,1.15)$ & 556 & 16.4 & 1.02 & $(0.92,1.12)$ & 6266 & 14.6 & 0.95 & $(0.92,0.9$ \\
\hline erchloroethylene & 29854 & 12.3 & 426 & 12.9 & 1.03 & $(0.92,1.15)$ & 413 & 12.2 & 1.01 & $(0.90,1.12)$ & 5308 & 12.4 & 1.02 & $(0.99,1.0$ \\
\hline Propylene dichloride & 1518 & 0.6 & 19 & 0.6 & 0.82 & $(0.52,1.29)$ & 33 & 1.0 & 1.39 & $(0.98,1.99)$ & 316 & 0.7 & 1.21 & $(1.0 \%, 1$ \\
\hline Tetrachloroethane & 1955 & 0.8 & 27 & 0.8 & 0.93 & $(0.63,1.37)$ & 33 & 1.0 & 1.09 & $(0.77,1.56)$ & 387 & 0.9 & 1.14 & $(1.02,1.2$ \\
\hline 1,1,2-trichloroethane & 2561 & 1.1 & 40 & 1.2 & 1.03 & $(0.75,1.42)$ & 48 & 1.4 & 1.19 & $(0.88,1.59)$ & 487 & 1.1 & 1.12 & $(1.01,1.2$ \\
\hline Trichloroethylene & 28547 & 11.8 & 409 & 12.4 & 0.98 & $(0.87,1.10)$ & 412 & 12.1 & 1.03 & $(0.92,1.15)$ & 5074 & 11.8 & 1.06 & $(1.02,1.10)$ \\
\hline 1,2,3-trichloropropane & 2847 & 1.2 & 44 & 1.3 & 1.01 & $(0.74,1.37)$ & 48 & 1.4 & 1.06 & $(0.79,1.42)$ & 542 & 1.3 & 1.13 & $(1.02,1.2$ \\
\hline
\end{tabular}

${ }^{a}$ Exposure risk value based on residential proximity to source(s) of air emissions and estimated pounds of chemical emitted annually.

${ }^{\mathrm{b}}$ Adjusted for birth year and maternal age, education, race/ethnicity, and public health region of residence (referent group: exposure risk value $=0$ ). 
Table 5 Maternal residential proximity to air emissions of chlorinated solvents and limb deficiency defects, Texas, 1996-2008

\begin{tabular}{|c|c|c|c|c|c|c|c|c|c|c|c|c|c|c|}
\hline \multirow{3}{*}{$\begin{array}{c}\text { Type of } \\
\text { chlorinated solvent }\end{array}$} & \multicolumn{14}{|c|}{ Exposure risk value $>0^{a}$} \\
\hline & \multicolumn{2}{|c|}{ Controls } & \multicolumn{2}{|c|}{$\begin{array}{c}\text { Any type of } \\
\text { limb deficiency }\end{array}$} & \multicolumn{2}{|c|}{$\begin{array}{l}\text { Adjusted odds } \\
\text { ratio }^{\text {b }}(95 \% \mathrm{Cl})\end{array}$} & \multicolumn{2}{|c|}{$\begin{array}{l}\text { Longitudinal } \\
\text { limb deficiency }\end{array}$} & \multicolumn{2}{|c|}{$\begin{array}{l}\text { Adjusted odds } \\
\text { ratio }^{\text {b }}(95 \% \mathrm{Cl})\end{array}$} & \multicolumn{2}{|c|}{$\begin{array}{c}\text { Transverse } \\
\text { limb deficiency }\end{array}$} & \multicolumn{2}{|c|}{ 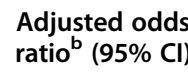 } \\
\hline & $n$ & $\%$ & $n$ & $\%$ & & & $n$ & $\%$ & & & $n$ & $\%$ & & \\
\hline Any type & 63599 & 26.2 & 568 & 28.7 & 1.05 & $(0.94,1.17)$ & 252 & 29.3 & 0.97 & $(0.82,1.14)$ & 334 & 27.7 & 1.09 & $(0.94,1.26)$ \\
\hline Carbon tetrachloride & 4568 & 1.9 & 39 & 2.0 & 1.07 & $(0.77,1.49)$ & 17 & 2.0 & 0.97 & $(0.59,1.61)$ & 21 & 1.7 & 1.01 & $(0.64,1.58)$ \\
\hline Chloroform & 3985 & 1.6 & 37 & 1.9 & 1.11 & $(0.79,1.57)$ & 16 & 1.9 & 1.04 & $(0.62,1.74)$ & 20 & 1.7 & 1.03 & $(0.65,1.63)$ \\
\hline 1,1-dichloroethane & 1847 & 0.8 & 14 & 0.7 & 1.00 & $(0.59,1.71)$ & 7 & 0.8 & 1.09 & $(0.51,2.32)$ & 7 & 0.6 & 0.87 & $(0.41$, \\
\hline 1,2-dichloroethane & 6637 & 2.7 & 52 & 2.6 & 0.98 & $(0.73,1.32)$ & 24 & 2.8 & 0.95 & $(0.62,1.46)$ & 29 & 2.4 & 0.96 & $(0.65,1.4)$ \\
\hline 1,2-dichloroethylene & 2026 & 0.8 & 17 & 0.9 & 1.09 & $(0.67,1.78)$ & 8 & 0.9 & 1.11 & $(0.54,2.26)$ & 8 & 0.7 & 0.89 & $(0.44,1.8$ \\
\hline Ethyl chloride & 5271 & 2.2 & 43 & 2.2 & 1.00 & $(0.73,1.37)$ & 20 & 2.3 & 1.01 & $(0.64,1.61)$ & 23 & 1.9 & 0.92 & $(0.60,1.41$ \\
\hline Methyl chloroform & 5294 & 2.2 & 46 & 2.3 & 0.94 & $(0.69,1.27)$ & 23 & 2.7 & 0.97 & $(0.63,1.48)$ & 23 & 1.9 & 0.82 & $(0.54,1.26$ \\
\hline Methylene chloride & 38448 & 15.9 & 361 & 18.2 & 1.06 & $(0.94,1.20)$ & 164 & 19.1 & 1.01 & $(0.84,1.22)$ & 204 & 16.9 & 1.04 & $(0.88,1.23$ \\
\hline Perchloroethylene & 29854 & 12.3 & 272 & 13.7 & 1.10 & $(0.96,1.26)$ & 113 & 13.2 & 0.95 & $(0.77,1.17)$ & 167 & 13.9 & 1.21 & $(1.01,1.4$ \\
\hline Propylene dichloride & 1518 & 0.6 & 13 & 0.7 & 1.15 & $(0.66,2.00)$ & 6 & 0.7 & 1.13 & $(0.50,2.56)$ & 6 & 0.5 & 0.93 & $(0.41,2.09)$ \\
\hline Tetrachloroethane & 1955 & 0.8 & 14 & 0.7 & 0.99 & $(0.58,1.69)$ & 6 & 0.7 & 0.93 & $(0.41,2.10)$ & 7 & 0.6 & 0.86 & $(0.40,1.83)$ \\
\hline 1,1,2-trichloroethane & 2561 & 1.1 & 18 & 0.9 & 0.91 & $(0.56,1.46)$ & 8 & 0.9 & 0.86 & $(0.42,1.75)$ & 9 & 0.7 & 0.79 & $(0.40,1.5$ \\
\hline Trichloroethylene & 28547 & 11.8 & 257 & 13.0 & 1.01 & $(0.88,1.17)$ & 119 & 13.9 & 1.00 & $(0.81,1.24)$ & 149 & 12.4 & 1.04 & $(0.86,1.26)$ \\
\hline 1,2,3-trichloropropane & 2847 & 1.2 & 24 & 1.2 & 1.10 & $(0.72,1.67)$ & 11 & 1.3 & 1.08 & $(0.58,1.99)$ & 12 & 1.0 & 0.95 & $(0.53,1.7$ \\
\hline
\end{tabular}

${ }^{a}$ Exposure risk value based on residential proximity to source(s) of air emissions and estimated pounds of chemical emitted annually.

${ }^{b}$ Adjusted for birth year and maternal age, education, race/ethnicity, and public health region of residence (referent group: exposure risk value $=0$ ).

propylene dichloride, tetrachloroethane, 1,1,2-trichloroethane, and 1,2,3-trichloropropane were observed in the highest categories of exposure risk values (Table 7). These trends were statistically significant $(p<0.05)$ for propylene dichloride and tetrachloroethane.

Positive linear trends were observed between septal heart defects and seven types of chlorinated solvents, including carbon tetrachloride; 1,1-dichloroethane; 1,2-dichloroethane; ethyl chloride; 1,1,2-trichloroethane; trichloroethylene; and 1,2,3-trichloropropane (Table 8). These associations tended to be weak with the ORs close to 1.00 , even in the highest exposure categories, and not monotonic with increasing intensities of exposure risk values, with the exception of odds ratios in relation to carbon tetrachloride emissions. We noted similar patterns of odds ratios and p-values for trend between categorization of exposure risk values into four (Table 8) or seven groups (data not shown), with the exception of all chlorinated solvents combined in which $p$-values for trend were 0.180 versus 0.035 respectively for the seven versus four levels of categorization. Significant linear trends were observed in the association between ethyl chloride emissions and obstructive heart defects (aOR in the highest exposure risk value $1.49,95 \%$ CI $1.09,2.05)$ and in the association between methylene chloride and isolated conotruncal heart defects (aOR in the highest exposure risk value 1.56, $95 \%$ CI 1.05, 2.32) (data not shown).
Associations between various chlorinated solvents and birth defects in offspring tended to be stronger among mothers 35 years or older than for younger mothers (Table 9). This pattern was most consistently observed with oral cleft defects and these solvents, including associations with any chlorinated solvent (older versus younger, aORs 1.22 versus 0.99 , respectively), carbon tetrachloride (aORs 1.66 versus 1.02), 1,1-dichloroethane (aORs 1.49 versus 0.85 ), 1,2-dichloroethane (aORs 1.50 versus 0.98 ), 1,2-dichloroethylene (aORs 1.65 versus 0.87 ), ethyl chloride (aORs 1.66 versus 1.05), methylene chloride (aORs 1.38 versus 1.02), tetrachloroethane (aORs 1.63 versus 0.85 ), 1,1,2-trichloroethane (aORs 1.78 versus 1.06 ), trichloroethylene (with cleft lip with or without cleft palate aORs 1.39 versus 1.02), and 1,2,3-trichloropropane (aORs 1.92 versus 1.12).

ORs for neural tube defects in offspring were also higher in older than younger mothers in relation to residential proximity to emissions of carbon tetrachloride (aORs 2.46 versus 1.31, respectively), chloroform (aORs 2.09 versus 1.33 ), and ethyl chloride (aORs 1.75 versus 1.36). On the other hand, this pattern was reversed for NTDs by maternal age in relation to 1,2-dichloroethane (younger versus older mothers, aORs 1.31 versus 0.98 , respectively). With respect to heart defects, associations were stronger for among older than younger women for residential proximity near trichloroethylene (with 
Table 6 Intensity of maternal residential exposure index for selected chlorinated solvents and spina bifida in offspring

\begin{tabular}{|c|c|c|c|c|c|c|c|c|}
\hline \multirow{2}{*}{$\begin{array}{c}\text { Type of } \\
\text { chlorinated solvent }\end{array}$} & \multirow{2}{*}{$\begin{array}{l}\text { Intensity of } \\
\text { exposure risk } \\
\text { value }^{\mathrm{a}}\end{array}$} & \multicolumn{2}{|c|}{ Spina bifida cases } & \multicolumn{2}{|c|}{ Controls } & \multirow{2}{*}{$\begin{array}{l}\text { Adjusted } \\
\text { odds } \\
\text { ratio }^{b}\end{array}$} & \multirow{2}{*}{$\begin{array}{c}95 \% \\
\text { confidence } \\
\text { interval }\end{array}$} & \multirow{2}{*}{$\begin{array}{c}p \text {-value } \\
\text { for } \\
\text { trend }\end{array}$} \\
\hline & & $n$ & $\%$ & $n$ & $\%$ & & & \\
\hline \multirow[t]{4}{*}{ Carbon tetrachloride } & 0.00 & 1269 & 96.6 & 237857 & 98.1 & 1.00 & Referent & 0.059 \\
\hline & $0.01-189.30$ & 18 & 1.4 & 1531 & 0.6 & 2.08 & $1.29,3.37$ & \\
\hline & $189.31-2342.12$ & 14 & 1.1 & 1531 & 0.6 & 1.53 & $0.89,2.64$ & \\
\hline & $>2342.12$ & 12 & 0.9 & 1506 & 0.6 & 1.19 & $0.66,2.14$ & \\
\hline \multirow[t]{4}{*}{ Chloroform } & 0.00 & 1276 & 97.2 & 238440 & 98.4 & 1.00 & Referent & 0.027 \\
\hline & $0.01-42.27$ & 14 & 1.1 & 1331 & 0.5 & 1.74 & $1.02,2.99$ & \\
\hline & $42.28-1490.26$ & 9 & 0.7 & 1331 & 0.5 & 1.23 & $0.63,2.40$ & \\
\hline & $>1490.26$ & 14 & 1.1 & 1323 & 0.5 & 1.66 & $0.94,2.91$ & \\
\hline \multirow[t]{4}{*}{ 1,1-dichloroethane } & 0.00 & 1294 & 98.6 & 240578 & 99.2 & 1.00 & Referent & 0.046 \\
\hline & $0.01-0.53$ & 8 & 0.6 & 618 & 0.3 & 2.18 & $1.07,4.43$ & \\
\hline & $0.54-6.59$ & 4 & 0.3 & 613 & 0.3 & 0.94 & $0.35,2.53$ & \\
\hline & $>6.59$ & 7 & 0.5 & 616 & 0.3 & 2.14 & $1.00,4.57$ & \\
\hline \multirow[t]{4}{*}{ 1,2-dichloroethane } & 0.00 & 1248 & 95.0 & 235788 & 97.3 & 1.00 & Referent & $<0.0001$ \\
\hline & $0.01-5.85$ & 17 & 1.3 & 2197 & 0.9 & 1.24 & $0.76,2.05$ & \\
\hline & $5.86-221.92$ & 24 & 1.8 & 2215 & 0.9 & 1.83 & $1.20,2.78$ & \\
\hline & $>221.92$ & 24 & 1.8 & 2225 & 0.9 & 1.85 & $1.21,2.84$ & \\
\hline \multirow[t]{4}{*}{ 1,2-dichloroethylene } & 0.00 & 1293 & 98.5 & 240399 & 99.2 & 1.00 & Referent & 0.065 \\
\hline & $0.01-0.48$ & 6 & 0.5 & 676 & 0.3 & 1.51 & $0.67,3.41$ & \\
\hline & $0.49-7.59$ & 8 & 0.6 & 677 & 0.3 & 1.79 & $0.88,3.63$ & \\
\hline & $>7.59$ & 6 & 0.5 & 673 & 0.3 & 1.49 & $0.66,3.36$ & \\
\hline \multirow[t]{4}{*}{ Ethyl chloride } & 0.00 & 1263 & 96.2 & 237154 & 97.8 & 1.00 & Referent & 0.003 \\
\hline & $0.01-12.11$ & 17 & 1.3 & 1761 & 0.7 & 1.71 & $1.04,2.81$ & \\
\hline & $12.12-98.74$ & 12 & 0.9 & 1764 & 0.7 & 1.17 & $0.65,2.08$ & \\
\hline & $>98.74$ & 21 & 1.6 & 1746 & 0.7 & 1.87 & $1.19,2.92$ & \\
\hline \multirow[t]{4}{*}{ Methyl chloroform } & 0.00 & 1256 & 95.7 & 237131 & 97.8 & 1.00 & Referent & 0.002 \\
\hline & $0.01-5.02$ & 21 & 1.6 & 1751 & 0.7 & 1.67 & $1.07,2.61$ & \\
\hline & 5.03-198.09 & 12 & 0.9 & 1778 & 0.7 & 1.08 & $0.60,1.92$ & \\
\hline & $>198.09$ & 24 & 1.8 & 1765 & 0.7 & 1.90 & $1.23,2.94$ & \\
\hline \multirow[t]{4}{*}{ Tetrachloroethane } & 0.00 & 1293 & 98.5 & 240470 & 99.2 & 1.00 & Referent & 0.033 \\
\hline & $0.01-0.18$ & 7 & 0.5 & 653 & 0.3 & 1.88 & $0.88,4.01$ & \\
\hline & $0.19-1.69$ & 7 & 0.5 & 653 & 0.3 & 1.83 & $0.86,3.92$ & \\
\hline & $>1.69$ & 6 & 0.5 & 649 & 0.3 & 1.62 & $0.72,3.66$ & \\
\hline \multirow[t]{4}{*}{ 1,1,2-trichloroethane } & 0.00 & 1283 & 97.7 & 239864 & 98.9 & 1.00 & Referent & 0.026 \\
\hline & $0.01-0.45$ & 15 & 1.1 & 853 & 0.4 & 3.01 & $1.77,5.10$ & \\
\hline & $0.46-1.78$ & 8 & 0.6 & 855 & 0.4 & 1.46 & $0.72,2.96$ & \\
\hline & $>1.78$ & 7 & 0.5 & 853 & 0.4 & 1.40 & $0.66,2.98$ & \\
\hline \multirow[t]{4}{*}{ 1,2,3-trichloropropane } & 0.00 & 1282 & 97.6 & 239578 & 98.8 & 1.00 & Referent & $<0.0001$ \\
\hline & $0.01-16.45$ & 8 & 0.6 & 950 & 0.4 & 1.31 & $0.65,2.68$ & \\
\hline & $16.46-132.51$ & 8 & 0.6 & 947 & 0.4 & 1.42 & $0.70,2.88$ & \\
\hline & $>132.51$ & 15 & 1.1 & 950 & 0.4 & 2.62 & $1.55,4.46$ & \\
\hline
\end{tabular}


Table 7 Intensity of maternal residential exposure index for selected chlorinated solvents and isolated cleft palate in offspring

\begin{tabular}{|c|c|c|c|c|c|c|c|c|}
\hline \multirow{2}{*}{$\begin{array}{c}\text { Type of } \\
\text { chlorinated solvent }\end{array}$} & \multirow{2}{*}{$\begin{array}{c}\text { Intensity of } \\
\text { exposure } \\
\text { risk value }^{\text {a }}\end{array}$} & \multicolumn{2}{|c|}{ Cleft palate cases } & \multicolumn{2}{|c|}{ Controls } & \multirow{2}{*}{$\begin{array}{l}\text { Adjusted } \\
\text { odds } \\
\text { ratio }\end{array}$} & \multirow{2}{*}{$\begin{array}{c}95 \% \\
\text { confidence } \\
\text { interval }\end{array}$} & \multirow{2}{*}{$\begin{array}{c}p \text {-value } \\
\text { for } \\
\text { trend }\end{array}$} \\
\hline & & $n$ & $\%$ & $n$ & $\%$ & & & \\
\hline \multirow[t]{4}{*}{ Propylene dichloride } & 0.00 & 1148 & 98.7 & 240907 & 99.4 & 1.00 & Referent & 0.031 \\
\hline & $0.01-0.02$ & 5 & 0.4 & 508 & 0.2 & 1.95 & $0.80,4.76$ & \\
\hline & $0.03-17.70$ & 3 & 0.3 & 506 & 0.2 & 1.03 & $0.33,3.25$ & \\
\hline & $>17.70$ & 7 & 0.6 & 504 & 0.2 & 2.32 & $1.09,4.95$ & \\
\hline \multirow[t]{4}{*}{ Tetrachloroethane } & 0.00 & 1146 & 98.5 & 240470 & 99.2 & 1.00 & Referent & 0.010 \\
\hline & $0.01-0.18$ & 3 & 0.3 & 653 & 0.3 & 0.90 & $0.29,2.82$ & \\
\hline & $0.19-1.69$ & 4 & 0.3 & 653 & 0.3 & 1.14 & $0.42,3.08$ & \\
\hline & $>1.69$ & 10 & 0.9 & 649 & 0.3 & 2.66 & $1.40,5.04$ & \\
\hline \multirow[t]{4}{*}{ 1,1,2-trichloroethane } & 0.00 & 1141 & 98.1 & 239864 & 98.9 & 1.00 & Referent & 0.059 \\
\hline & $0.01-0.45$ & 8 & 0.7 & 853 & 0.4 & 1.66 & $0.82,3.37$ & \\
\hline & $0.46-1.78$ & 4 & 0.3 & 855 & 0.4 & 0.84 & $0.31,2.27$ & \\
\hline & $>1.78$ & 10 & 0.9 & 853 & 0.4 & 1.99 & $1.05,3.76$ & \\
\hline \multirow[t]{4}{*}{ 1,2,3-trichloropropane } & 0.00 & 1139 & 97.9 & 239578 & 98.8 & 1.00 & Referent & 0.083 \\
\hline & $0.01-16.45$ & 10 & 0.9 & 950 & 0.4 & 1.77 & $0.94,3.37$ & \\
\hline & $16.46-132.51$ & 4 & 0.3 & 947 & 0.4 & 0.74 & $0.28,2.00$ & \\
\hline & $>132.51$ & 10 & 0.9 & 950 & 0.4 & 1.91 & $1.01,3.63$ & \\
\hline
\end{tabular}

${ }^{a}$ Exposure risk value based on maternal residential proximity to source(s) of air emissions and estimated pounds of chemical emitted annually.

${ }^{b}$ Adjusted for birth year and maternal race/ethnicity, age, education, and public health region.

obstructive heart defects aORs 1.43 versus 0.97 ) and any chlorinated solvent emissions (with obstructive heart defects aORs 1.27 versus 0.98 ). In contrast, associations were stronger for younger mothers than older mothers for the relation between ethyl chloride and atrioventricular septal defects, obstructive heart defects, and septal heart defects in offspring. While only one set of associations with higher odds ratios in younger versus older mothers showed significant multiplicative interaction, 16 sets of the stronger associations in older versus younger mothers showed significant additive and/or multiplicative interaction by maternal age. Too few older casemothers of babies with limb deficiencies were available to assess interaction by maternal age in this birth defect group.

\section{Discussion}

In this large population-based, case-control study, casemothers (of offspring with neural tube defects, oral cleft defects, and congenital heart defects) were more likely than control-mothers to have higher estimated exposures to industrial air emissions of chlorinated solvents, based on their residential distance to such facilities and reported annual releases. Associations between maternal residential proximity to these emissions and birth defects in offspring tended to be stronger among mothers who were 35 years or older than among younger mothers, especially with oral cleft defects.
Other studies of the relation between maternal occupational and environmental exposures to chlorinated solvents have indicated associations between these solvents and various birth defects. In the NBDPS population, maternal occupational exposure to chlorinated solvents during the periconceptional period was strongly associated with neural tube defects (aOR 1.96, 95\% CI 1.34, 2.97), especially spina bifida (aOR 2.26, 95\% CI 1.44, 3.53) [12]. Odds ratios for specific chlorinated solvents were not reported, although the prevalence of occupational exposures to chloroform, methylene chloride, perchloroethylene, trichloroethane, and trichloroethylene were all higher among case-mothers of babies with spina bifida than control mothers. In the present study, case-mothers of babies with spina bifida were also more likely than control-mothers to live near industrial emissions of chloroform, methylene chloride, trichloroethane, and trichloroethylene, but the same proportion of case-mothers as control-mothers lived near industrial emissions of perchloroethylene.

In two French study populations, maternal occupational exposures to chlorinated solvents [11] or halogenated aliphatic solvents [22] (also known as alkyl halide solvents of which chlorinated solvents are a subgroup) were associated with oral cleft defects in offspring, specifically with cleft lip with or without cleft palate. These findings were not corroborated in a U.S. study population in which the odds for any oral cleft, cleft palate, and cleft lip with or without cleft palate in offspring in 
Table 8 Intensity of maternal residential exposure index for selected chlorinated solvents and septal heart defects in offspring

\begin{tabular}{|c|c|c|c|c|c|c|c|c|}
\hline \multirow{2}{*}{$\begin{array}{c}\text { Type of } \\
\text { chlorinated solvent }\end{array}$} & \multirow{2}{*}{$\begin{array}{c}\text { Intensity of } \\
\text { exposure risk } \\
\text { value }^{a}\end{array}$} & \multicolumn{2}{|c|}{ Septal heart defect cases } & \multicolumn{2}{|c|}{ Controls } & \multirow{2}{*}{$\begin{array}{c}\text { Adjusted } \\
\text { odds } \\
\text { ratio }^{b}\end{array}$} & \multirow{2}{*}{$\begin{array}{c}95 \% \\
\text { confidence } \\
\text { interval }\end{array}$} & \multirow{2}{*}{$\begin{array}{c}p \text {-value } \\
\text { for } \\
\text { trend }\end{array}$} \\
\hline & & $n$ & $\overline{\%}$ & $n$ & $\overline{\%}$ & & & \\
\hline \multirow[t]{4}{*}{ Any type } & 0.00 & 31536 & 73.6 & 178826 & 73.8 & 1.00 & Referent & 0.035 \\
\hline & $0.01-296.24$ & 4196 & 9.8 & 21301 & 8.8 & 1.14 & $1.10,1.18$ & \\
\hline & $296.25-3457.84$ & 3542 & 8.3 & 21191 & 8.7 & 1.00 & $0.96,1.04$ & \\
\hline & $>3457.84$ & 3587 & 8.4 & 21107 & 8.7 & 1.03 & $0.99,1.08$ & \\
\hline \multirow[t]{4}{*}{ Carbon tetrachloride } & 0.00 & 41994 & 98.0 & 237857 & 98.1 & 1.00 & Referent & $<0.0001$ \\
\hline & $0.01-189.30$ & 271 & 0.6 & 1531 & 0.6 & 1.03 & $0.90,1.18$ & \\
\hline & 189.31-2342.12 & 301 & 0.7 & 1531 & 0.6 & 1.14 & $1.01,1.30$ & \\
\hline & $>2342.12$ & 295 & 0.7 & 1506 & 0.6 & 1.21 & $1.07,1.38$ & \\
\hline \multirow[t]{4}{*}{ 1,1-dichloroethane } & 0.00 & 42474 & 99.1 & 240578 & 99.2 & 1.00 & Referent & 0.003 \\
\hline & $0.01-0.53$ & 139 & 0.3 & 618 & 0.3 & 1.32 & $1.09,1.59$ & \\
\hline & $0.54-6.59$ & 121 & 0.3 & 613 & 0.3 & 1.18 & $0.97,1.44$ & \\
\hline & $>6.59$ & 127 & 0.3 & 616 & 0.3 & 1.18 & $0.97,1.43$ & \\
\hline \multirow[t]{4}{*}{ 1,2-dichloroethane } & 0.00 & 41677 & 97.2 & 235788 & 97.3 & 1.00 & Referent & 0.007 \\
\hline & $0.01-5.85$ & 350 & 0.8 & 2197 & 0.9 & 0.96 & $0.86,1.08$ & \\
\hline & $5.86-221.92$ & 384 & 0.9 & 2215 & 0.9 & 1.03 & $0.92,1.15$ & \\
\hline & $>221.92$ & 450 & 1.0 & 2225 & 0.9 & 1.19 & $1.07,1.32$ & \\
\hline \multirow[t]{4}{*}{ 1,2-dichloroethylene } & 0.00 & 42452 & 99.0 & 240399 & 99.2 & 1.00 & Referent & 0.079 \\
\hline & $0.01-0.48$ & 165 & 0.4 & 676 & 0.3 & 1.42 & $1.20,1.69$ & \\
\hline & $0.49-7.59$ & 127 & 0.3 & 677 & 0.3 & 1.11 & $0.92,1.35$ & \\
\hline & $>7.59$ & 117 & 0.3 & 673 & 0.3 & 1.02 & $0.84,1.24$ & \\
\hline \multirow[t]{4}{*}{ Ethyl chloride } & 0.00 & 41859 & 97.7 & 237154 & 97.8 & 1.00 & Referent & 0.004 \\
\hline & $0.01-12.11$ & 335 & 0.8 & 1761 & 0.7 & 1.11 & $0.98,1.25$ & \\
\hline & $12.12-98.74$ & 360 & 0.8 & 1764 & 0.7 & 1.19 & $1.06,1.34$ & \\
\hline & $>98.74$ & 307 & 0.7 & 1746 & 0.7 & 1.08 & $0.95,1.22$ & \\
\hline \multirow[t]{4}{*}{ Perchloroethylene } & 0.00 & 37553 & 87.6 & 212571 & 87.7 & 1.00 & Referent & 0.045 \\
\hline & $0.01-41.59$ & 2131 & 5.0 & 9990 & 4.1 & 1.19 & $1.13,1.25$ & \\
\hline & $41.60-221.22$ & 1605 & 3.7 & 9959 & 4.1 & 0.94 & $0.89,0.99$ & \\
\hline & $>221.22$ & 1572 & 3.7 & 9905 & 4.1 & 0.92 & $0.87,0.97$ & \\
\hline \multirow[t]{4}{*}{ Propylene dichloride } & 0.00 & 42545 & 99.3 & 240907 & 99.4 & 1.00 & Referent & 0.143 \\
\hline & $0.01-0.02$ & 135 & 0.3 & 508 & 0.2 & 1.55 & $1.28,1.88$ & \\
\hline & $0.03-17.70$ & 101 & 0.2 & 506 & 0.2 & 1.15 & $0.93,1.43$ & \\
\hline & $>17.70$ & 80 & 0.2 & 504 & 0.2 & 0.94 & $0.74,1.19$ & \\
\hline \multirow[t]{4}{*}{ Tetrachloroethane } & 0.00 & 42474 & 99.1 & 240470 & 99.2 & 1.00 & Referent & 0.113 \\
\hline & $0.01-0.18$ & 149 & 0.3 & 653 & 0.3 & 1.32 & $1.10,1.58$ & \\
\hline & $0.19-1.69$ & 114 & 0.3 & 653 & 0.3 & 1.00 & $0.82,1.23$ & \\
\hline & $>1.69$ & 124 & 0.3 & 649 & 0.3 & 1.11 & $0.91,1.24$ & \\
\hline \multirow[t]{4}{*}{ 1,1,2-trichloroethane } & 0.00 & 42374 & 98.9 & 239864 & 98.9 & 1.00 & Referent & 0.031 \\
\hline & $0.01-0.45$ & 148 & 0.3 & 853 & 0.4 & 1.04 & $0.87,1.24$ & \\
\hline & $0.46-1.78$ & 181 & 0.4 & 855 & 0.4 & 1.25 & $1.06,1.47$ & \\
\hline & $>1.78$ & 158 & 0.4 & 853 & 0.4 & 1.08 & $0.91,1.28$ & \\
\hline
\end{tabular}


Table 8 Intensity of maternal residential exposure index for selected chlorinated solvents and septal heart defects in offspring (Continued)

\begin{tabular}{|c|c|c|c|c|c|c|c|c|}
\hline \multirow[t]{4}{*}{ Trichloroethylene } & 0.00 & 37787 & 88.2 & 213878 & 88.2 & 1.00 & Referent & 0.002 \\
\hline & $0.01-56.69$ & 1718 & 4.0 & 9539 & 3.9 & 1.07 & $1.01,1.13$ & \\
\hline & $56.70-284.39$ & 1611 & 3.8 & 9485 & 3.9 & 1.02 & $0.96,1.08$ & \\
\hline & $>284.39$ & 1745 & 4.1 & 9523 & 3.9 & 1.09 & $1.03,1.15$ & \\
\hline \multirow[t]{4}{*}{ 1,2,3-trichloropropane } & 0.00 & 42319 & 98.7 & 239578 & 98.8 & 1.00 & Referent & 0.033 \\
\hline & $0.01-16.45$ & 168 & 0.4 & 950 & 0.4 & 1.05 & $0.89,1.24$ & \\
\hline & $16.46-132.51$ & 210 & 0.5 & 947 & 0.4 & 1.31 & $1.12,1.52$ & \\
\hline & $>132.51$ & 164 & 0.4 & 950 & 0.4 & 1.02 & $0.86,1.21$ & \\
\hline
\end{tabular}

${ }^{a}$ Exposure risk value based on maternal residential proximity to source(s) of air emissions and estimated pounds of chemical emitted annually.

${ }^{\mathrm{b}}$ Adjusted for birth year and maternal race/ethnicity, age, education, and public health region.

relation to maternal occupational exposures to chlorinated solvents were close to 1.00 [12]. With respect to environmental exposures, case-mothers of babies were oral clefts were not more likely than control- mothers in Texas to live within a mile of an industrial facility with emissions of alkyl halide solvents [16]. In the present study, few associations were noted between maternal proximity to emissions of specific chlorinated solvents and oral clefts, except for cleft palate with propylene dichloride, tetrachloroethane, and 1,1,2-trichloroethane. With stratification by maternal age, however, proximity to chlorinated solvent emissions overall was significantly associated with any oral cleft and specifically with cleft lip with or without cleft palate among mothers 35 years or older. Stronger associations were observed among older mothers between residential proximity to several types of chlorinated solvent emissions and cleft palate alone and cleft lip with or without cleft palate in offspring.

In a study conducted in France in which metabolites of chlorinated solvents were measured in urine, case-mothers of babies with limb malformations were more likely than control mothers to have higher levels of urinary metabolites of tetrachloroethylene (perchloroethylene) and trichloroethylene [13]. We also observed an association between maternal proximity to emissions of perchloroethylene and transverse limb defects in offspring, but the odds ratios were close to 1.0 for associations between trichloroethylene emissions and limb deficiencies in our study.

With respect to heart defects, maternal occupational exposures to chlorinated solvents were associated with perimembranous ventricular septal defects (aOR 1.7, 95\% CI 1.0, 2.8) in the National Birth Defects Study population; other associations were noted with d-transposition of the great arteries and aortic stenosis although the 95\% confidence intervals were compatible with the null [14]. In a study of maternal residential proximity to industrial facilities and selected birth defects in offspring in Texas, conotruncal heart defects were not associated with maternal residential proximity to facilities reporting air emissions of alkyl halide solvents [17]. In the present study, we also did not observe any associations with conotruncal heart defects with chlorinated solvent emissions, and most of the significant associations with these solvents were restricted to septal heart defects. However, among older mothers, obstructive heart defects in offspring were associated with maternal proximity to emissions of any chlorinated solvents and specifically with emissions of ethyl chloride and trichloroethylene. Older mothers of offspring with any type of heart defect and septal heart defects were also more likely than older control-mothers to live near emissions of trichloroethylene.

Maternal age has been found in other studies to modify associations between maternal prenatal exposures to chemicals and pregnancy outcomes. In a study of trichloroethylene-emitting sites and congenital heart defects in Wisconsin, older case-mothers (38 years or older) of offspring with congenital heart defects were over three times more likely than older control-mothers to live within 1.32 miles of trichloroethylene-emitting sites; no association between congenital heart defects and this residential characteristic was noted among offspring of younger women [15]. Infants of mothers 35 years or older who were exposed to tetrachloroethylene in drinking water had lower mean birth weights and were more likely to be small for gestational age than infants among unexposed women in the same age group in a study conducted at a U.S. Marine Corps base in North Carolina; these patterns were not observed in offspring of younger mothers [23]. The study investigators suggested that advanced maternal age might increase susceptibility of the developing fetus to chemical insults.

In the present study, we used maternal residential addresses at delivery to determine residential proximity to emissions of chlorinated solvents. While maternal address at delivery corresponds to maternal address during the first trimester for most mothers, results from two previous studies of Texas case-mothers and controlmothers who participated in the National Birth Defects 
Table 9 Maternal residential proximity to air emissions of chlorinated solvents and birth defects by maternal age

\begin{tabular}{|c|c|c|c|c|c|c|c|c|c|}
\hline \multirow[t]{4}{*}{$\begin{array}{c}\text { Type of chlorinated } \\
\text { solvent }\end{array}$} & \multirow[t]{4}{*}{ Birth defect group } & \multirow{2}{*}{\multicolumn{8}{|c|}{$\begin{array}{c}\text { Number (\%) with residential exposure risk value }>0 \text {, adjusted odds ratios (OR), } \\
\text { and } 95 \% \text { confidence intervals }(\mathrm{Cl}) \\
\text { Maternal age group }\end{array}$}} \\
\hline & & & & & & & & & \\
\hline & & \multicolumn{4}{|c|}{$<35$ years } & \multicolumn{4}{|c|}{35 years or older } \\
\hline & & Number & (\%) & $\mathrm{OR}^{\mathrm{a}}$ & $(95 \% \mathrm{Cl})$ & Number & $(\%)$ & $O^{a}$ & $(95 \% \mathrm{Cl})$ \\
\hline \multirow[t]{4}{*}{ Any chlorinated solvent } & Control births & 56735 & $(26.2)$ & - & - & 6864 & $(26.3)$ & - & - \\
\hline & Any type of oral cleft defect & 1478 & $(27.3)$ & 0.99 & $(0.92,1.06)$ & 240 & $(30.6)$ & 1.22 & $(1.03,1.46)^{b}$ \\
\hline & Cleft lip with or without cleft palate & 934 & $(26.7)$ & 0.97 & $(0.89,1.06)$ & 161 & $(33.0)$ & 1.37 & $(1.10,1.70)^{b, c}$ \\
\hline & Obstructive heart defects & 767 & $(26.2)$ & 0.98 & $(0.89,1.08)$ & 145 & $(31.0)$ & 1.27 & $(1.01,1.58)^{b, c}$ \\
\hline \multirow[t]{6}{*}{ Carbon tetrachloride } & Control births & 4195 & $(1.9)$ & - & - & 373 & $(1.4)$ & - & - \\
\hline & Any type of neural tube defect & 52 & $(2.6)$ & 1.31 & $(0.98,1.75)$ & 10 & $(4.7)$ & 2.46 & $(1.23,4.91)^{\mathrm{b}, \mathrm{c}}$ \\
\hline & Spina bifida & 37 & $(3.2)$ & 1.46 & $(1.03,2.07)$ & 7 & $(4.9)$ & 2.49 & $(1.09,5.72)^{b}$ \\
\hline & Any type of oral cleft defect & 122 & $(2.3)$ & 1.02 & $(0.85,1.24)$ & 21 & $(2.7)$ & 1.66 & $(1.04,2.65)^{b, c}$ \\
\hline & Cleft palate alone & 47 & $(2.4)$ & 1.05 & $(0.77,1.42)$ & 8 & $(2.7)$ & 1.76 & $(0.84,3.72)$ \\
\hline & Cleft lip with or without cleft palate & 75 & $(2.1)$ & 1.01 & $(0.79,1.28)$ & 13 & $(2.7)$ & 1.59 & $(0.88,2.86)$ \\
\hline \multirow[t]{3}{*}{ Chloroform } & Control births & 3662 & $(1.7)$ & - & - & 323 & $(1.2)$ & - & - \\
\hline & Any type of neural tube defect & 45 & $(2.3)$ & 1.33 & $(0.97,1.81)$ & 7 & (3.3) & 2.09 & $(0.93,4.68)$ \\
\hline & Spina bifida & 32 & $(2.7)$ & 1.48 & $(1.02,2.14)$ & 5 & (3.5) & 2.16 & $(0.82,5.67)$ \\
\hline \multirow[t]{2}{*}{ 1,1-dichloroethane } & Control births & 1704 & $(0.8)$ & - & - & 143 & $(0.5)$ & - & - \\
\hline & Any type of oral cleft defect & 40 & $(0.7)$ & 0.85 & $(0.62,1.17)$ & 7 & $(0.9)$ & 1.49 & $(0.69,3.25)$ \\
\hline \multirow[t]{5}{*}{ 1,2-dichloroethane } & Control births & 6033 & $(2.8)$ & - & - & 604 & $(2.3)$ & - & - \\
\hline & Any type of neural tube defect & 78 & $(4.0)$ & 1.31 & $(1.02,1.69)$ & 7 & (3.3) & 0.98 & $(0.44,2.16)$ \\
\hline & Spina bifida & 60 & $(5.1)$ & 1.70 & $(1.27,2.28)$ & 5 & $(3.5)$ & 1.09 & $(0.42,2.80)$ \\
\hline & Any type of oral cleft defect & 168 & (3.1) & 0.98 & $(0.83,1.16)$ & 29 & $(3.7)$ & 1.50 & $(1.00,2.26)^{b}$ \\
\hline & Cleft palate alone & 64 & 3.3 & 1.00 & $(0.77,1.31)$ & 13 & $(4.3)$ & 1.93 & $(1.05,3.54)^{b}$ \\
\hline \multirow[t]{3}{*}{ 1,2-dichloroethylene } & Control births & 1862 & $(0.9)$ & - & - & 164 & $(0.6)$ & - & - \\
\hline & Any type of oral cleft defect & 45 & $(0.8)$ & 0.87 & $(0.64,1.17)$ & 9 & (1.1) & 1.65 & $(0.83,3.30)$ \\
\hline & Cleft palate alone & 15 & $(0.8)$ & 0.75 & $(0.45,1.26)$ & 5 & $(1.7)$ & 2.54 & $(1.00,6.43)^{b, c}$ \\
\hline \multirow[t]{10}{*}{ Ethyl chloride } & Control births & 4820 & $(2.2)$ & - & - & 451 & $(1.7)$ & - & - \\
\hline & Any type of neural tube defect & 62 & $(3.2)$ & 1.36 & $(1.04,1.77)$ & 8 & (3.7) & 1.75 & $(0.83,3.68)$ \\
\hline & Spina bifida & 43 & $(3.7)$ & 1.48 & $(1.08,2.05)$ & 7 & $(4.9)$ & 2.50 & $(1.11,5.63)$ \\
\hline & Any type of oral cleft defect & 144 & $(2.7)$ & 1.05 & $(0.88,1.25)$ & 24 & $(3.1)$ & 1.66 & $(1.07,2.56)^{b}$ \\
\hline & Cleft palate alone & 52 & $(2.7)$ & 1.01 & $(0.76,1.34)$ & 8 & $(2.7)$ & 1.41 & $(0.68,2.94)$ \\
\hline & Cleft lip with or without cleft palate & 92 & $(2.6)$ & 1.08 & $(0.87,1.34)$ & 16 & $(3.3)$ & 1.81 & $(1.06,3.07)^{b}$ \\
\hline & Any type of heart defect & 1065 & $(2.4)$ & 1.14 & $(1.07,1.23)$ & 143 & $(1.8)$ & 0.99 & $(0.81,1.20)$ \\
\hline & Atrioventricular septal defect & 43 & (3.8) & 1.39 & $(1.01,1.91)$ & 4 & $(1.0)$ & 0.58 & $(0.21,1.59)^{c}$ \\
\hline & Obstructive heart defect & 92 & (3.1) & 1.29 & $(1.04,1.61)$ & 6 & $(1.3)$ & 0.63 & $(0.28,1.44)$ \\
\hline & Septal heart defect & 883 & $(2.4)$ & 1.14 & $(1.06,1.23)$ & 119 & $(1.8)$ & 0.98 & $(0.79,1.20)$ \\
\hline \multirow[t]{3}{*}{ Methylene chloride } & Control births & 34553 & $(16.0)$ & - & - & 3895 & $(14.0)$ & - & - \\
\hline & Any type of oral cleft defect & 942 & $(17.4)$ & 1.02 & $(0.94,1.10)$ & 158 & $(20.2)$ & 1.38 & $(1.14,1.67)^{b, c}$ \\
\hline & Cleft lip with or without cleft palate & 603 & $(17.3)$ & 1.02 & $(0.92,1.12)$ & 107 & $(21.9)$ & 1.53 & $(1.21,1.93)^{b, c}$ \\
\hline \multirow[t]{3}{*}{ Tetrachloroethane } & Control births & 1801 & $(0.8)$ & - & - & 154 & $(0.6)$ & - & - \\
\hline & Any type of oral cleft defect & 41 & $(0.8)$ & 0.85 & $(0.62,1.16)$ & 8 & $(1.0)$ & 1.63 & $(0.79,3.39)$ \\
\hline & Cleft lip with or without cleft palate & 24 & $(0.7)$ & 0.79 & $(0.53,1.20)$ & 5 & $(1.0)$ & 1.57 & $(0.63,3.93)$ \\
\hline
\end{tabular}


Table 9 Maternal residential proximity to air emissions of chlorinated solvents and birth defects by maternal age (Continued)

\begin{tabular}{|c|c|c|c|c|c|c|c|c|c|}
\hline \multirow[t]{3}{*}{ 1,1,2-trichloroethane } & Control births & 2354 & $(1.1)$ & - & - & 207 & (0.8) & - & - \\
\hline & Any type of oral cleft defect & 70 & $(1.3)$ & 1.06 & $(0.83,1.35)$ & 12 & (1.5) & 1.78 & $(0.97,3.25)$ \\
\hline & Cleft palate alone & 26 & $(1.4)$ & 1.03 & $(0.69,1.53)$ & 5 & $(1.7)$ & 2.06 & $(0.82,5.19)$ \\
\hline \multirow[t]{5}{*}{ Trichloroethylene } & Control births & 25432 & (11.8) & - & - & 3124 & (12.0) & - & - \\
\hline & Cleft lip with or without cleft palate & 439 & (12.6) & 1.02 & $(0.91,1.14)$ & 78 & $(16.0)$ & 1.39 & $(1.06,1.83)^{\mathrm{b}, \mathrm{c}}$ \\
\hline & Any type of heart defect & 5067 & (11.6) & 1.04 & $(1.00,1.07)$ & 1025 & (12.6) & 1.13 & $(1.04,1.22)$ \\
\hline & Obstructive heart defects & 338 & (11.6) & 0.97 & $(0.86,1.10)$ & 74 & $(15.8)$ & 1.43 & $(1.08,1.88)^{\mathrm{b}, \mathrm{c}}$ \\
\hline & Septal heart defects & 4217 & $(11.7)$ & 1.04 & $(1.01,1.08)$ & 857 & $(12.7)$ & 1.13 & $(1.03,1.23)$ \\
\hline \multirow[t]{4}{*}{ 1,2,3-trichloropropane } & Control births & 2602 & $(1.2)$ & - & - & 241 & (0.9) & - & - \\
\hline & Any type of oral cleft defect & 83 & $(1.5)$ & 1.12 & $(0.90,1.41)$ & 15 & (1.9) & 1.92 & $(1.11,3.32)^{b}$ \\
\hline & Cleft palate alone & 34 & (1.8) & 1.22 & $(0.86,1.74)$ & 6 & (2.0) & 2.11 & $(0.90,4.94)$ \\
\hline & Cleft lip with or without cleft palate & 49 & (1.4) & 1.07 & $(0.79,1.43)$ & 9 & $(1.8)$ & 1.80 & $(0.90,3.60)$ \\
\hline
\end{tabular}

${ }^{a}$ Odds ratios adjusted for year of birth and maternal education, race/ethnicity, and public health region of residence.

${ }^{\text {b}}$ Statistically significant additive interaction by maternal age group.

${ }^{c}$ Statistically significant multiplicative interaction by maternal age group.

Prevention Study indicated that up to $33 \%$ of the casemothers and $31 \%$ of the control-mothers changed residence between conception and delivery $[24,25]$. Therefore, some mothers in this study may have been misclassified with respect to residential proximity to emissions of chlorinated solvents during the first trimester, the relevant period of morphogenesis of the birth defects included in this study. On the other hand, older mothers in Texas were observed to move less during pregnancy than younger mothers with approximately $15 \%$ of the case- and control-mothers, who were 30 years or older, changing residences between conception and delivery [24]. Furthermore, maternal residential movement of mothers in all age categories tended to involve short distances [25] which would have minimal impact on exposure assessment to air pollutants.

Sufficient address information was not available to geocode all maternal addresses to street level, although proportions geocoded to this level included approximately $87 \%$ of the control group addresses and 84 to $87 \%$ of case group addresses, with the exception of neural tube defects with $69.1 \%$ geocoded to the street level. Geocoded and ungeocoded controls and case groups (heart, oral cleft, limb reduction, and neural tube defect groups) had similar distributions by maternal age, but the ungeocoded case groups and controls had higher proportions of mothers who were Hispanic and who had less than a high school education than the respective geocoded groups. Given that the differences were in the same direction and similar in magnitude among case and control groups, it is most likely that a compensating bias occurred (both cases and controls had the bias in the same direction and of similar magnitude). Therefore, we might expect that the exclusion of the ungeocoded cases and controls to have minimal impact on the odds ratios other than reducing their precision due to reduced sample sizes. Approximately $10 \%$ of industrial facilities also could not be geocoded because of insufficient and/ or inaccurate address information. These missing industries may have led to misclassification of exposure status for some case and control residences, although it is not likely that this misclassification would have been differential with respect to case or control status.

As a proxy for potential exposure to emissions of chlorinated solvents, we used a metric (EWPM) that incorporated residential distance from all TRI facilities within a $10 \mathrm{~km}$ radius that reported air emissions of chlorinated solvents and annual amounts of chemicals reported released from each facility. This approach probably introduced some misclassification of exposures. To assess the performance of EWPM in predicting the presence and intensity of chemical air emissions, we examined how well the EWPM exposure metrics correlated with air measurements of chlorinated solvents taken by the Texas Commission of Environmental Quality at 48 monitoring sites in Texas during 2005. The EWPM measurements were positively correlated with air measurements for the six chemicals available for comparison, including carbon tetrachloride, chloroform, 1,1-dichloroethane, methyl chloroform, 1,1,2-trichloroethane, and trichloroethylene, with the strongest correlations noted with carbon tetrachloride and chloroform [unpublished observations, Gong and Zhan]. In addition to the limitation of chemical quantities aggregated into pounds per year, further misclassification could have been introduced if the amounts released per year varied since the first trimester of some pregnancies might have been during the preceding year. Furthermore, length of gestation for fetal deaths and induced terminations 
(cases) would have been shorter than that for the majority of live births, although approximately $97 \%$ of the cases were liveborn and the most vulnerable period for teratogenesis is the first trimester.

While the Texas Birth Defects Registry includes elective terminations in their active surveillance of birth defects, we were unable to include these cases in this study because of missing data from lack of linkage to a vital record. This lack of linkage had the greatest impact on our analyses of the relation between chlorinated solvent emissions and neural tube defects in which $69 \%$ of maternal addresses were geocoded to street level and $22 \%$ were missing information regarding maternal education, a variable strongly related to maternal residential proximity to industrial facilities along with maternal race/ ethnicity [26].

With respect to specific defects, the highest proportion of induced terminations were found with anencephalic cases (39.7\%) with percentages less than 5\% for the other studied defects with the exception of spina bifida (8.3\%) and lower limb reduction defects (5.6\%). Given the high proportion of anencephalic cases that could not be analyzed because of missing information, findings related to this defect should be interpreted cautiously because it is not known whether women who undergo induced terminations are more or less likely to live near sources of industrial emissions.

In this study, we were unable to account for recurrence of birth defects in subsequent pregnancies of case-women, a known risk factor for birth defects [27]. Recurrence would most likely introduce bias into the study if it was related to maternal residence as well as prevalence of birth defects. We are not aware of any studies that document a higher or lower likelihood of mothers living near industrial sites with chemical emissions if they have had a previous birth defect-affected pregnancy. On the other hand, while Lie et al. [28] noted less than a 5\% recurrence of birth defects in Norwegian women whose first infants had birth defects, women who resided in the same municipality during both pregnancies were 11.6 times more likely than women whose first infant had no defect, to have a second infant with the same defect. In contrast, women who moved to another municipality after the birth of their first infant were 5.1 times more likely to have a second infant with the same defect. The authors concluded that these findings suggested that environmental factors contribute to the familial risk of birth defects and that important environmental teratogens have yet to be discovered.

\section{Conclusions}

The large sample size of this study allowed examination of associations between specific chlorinated solvents and birth defects as well as stratification by maternal age. Furthermore, a refined exposure assessment accounted for multiple facilities and their respective annual quantities of chemical releases, an improvement from previous studies that estimated exposure based on distance to the nearest industrial facility.

Study findings suggest that maternal residential proximity to air emissions of chlorinated solvents are associated with neural tube, oral cleft, and congenital heart defects, especially among offspring of older mothers. To better understand the relation between maternal exposure to chlorinated solvent emissions and birth defects in offspring, we recommend that future studies focus on populations in which air measurements of these chemicals and residential histories during pregnancy are available.

\section{Abbreviations}

aOR: Adjusted odds ratio; Cl: Confidence interval; EWPM: Emission Weighted Proximity Model; NBDPS: National Birth Defects Prevention Study;

NTD: Neural tube defects; OR: Odds ratio; TBDR: Texas Birth Defect Registry; TCE: Trichloroethylene; TRI: Toxic Release Inventory; USEPA: United States Environmental Protection Agency.

\section{Competing interests}

The authors declare that they have no competing interests.

\section{Authors' contributions}

$J B$ (in conjunction with FZ) conceived of the study, participated in the study design and data analyses, drafted the discussion section, and coordinated the final draft of the manuscript. MS carried out the data analyses and wrote the initial draft of the manuscript. FZ supervised and participated in the geocoding of industrial sites, the linkage of industrial sites and releases to maternal addresses, the development of exposure risk values, and the writing of the environmental sections of the manuscript. XG participated in the geocoding of industrial sites, the linkage of industrial sites and releases to maternal addresses, and performed the correlation analyses between exposure risk values and air monitoring data. PH supplied the birth defect case and control databases, participated in various aspects of study design and data analysis, and the drafting of the manuscript. All authors read and approved the final manuscript.

\section{Acknowledgements}

The research reported in this paper was made possible in part by a USEPA-STAR grant (\#R834790). The contents are solely the responsibility of the authors and do not necessarily represent the official views of the USEPA. Furthermore, USEPA and the authors do not endorse the purchase of any commercial products or services mentioned in the poster. This publication was also supported in part through a cooperative agreement

(U01DD000494) between the Centers for Disease Control and Prevention and the Texas Department of State Health Services (DSHS) and by Title $\mathrm{V}$ Maternal and Child Health Block Grants Funds from the Office of Title $V$ and Family Health, Texas DSHS. Its contents are solely the responsibility of the authors and do not necessarily represent the official views of the Centers for Disease Control and Prevention. Some of the data used in the analyses were provided by the Texas Department of State Health Services (DSHS) Birth Defects Epidemiology and Surveillance Branch and the DSHS Center for Health Statistics. The authors express their appreciation to the USEPA and Texas Department of State Health Services.

\section{Author details}

${ }^{1}$ Texas A\&M Health Science Center School of Public Health, Department of Epidemiology \& Biostatistics, College Station, TX 77843-1266, USA.

2Department of Geography, Texas Center for Geographic Information Science, Texas State University, San Marcos, TX 78666, USA. ${ }^{3}$ Birth Defects Epidemiology and Surveillance Branch, Texas Department of State Health Services, PO Box 149347 MC 1964, Austin, TX 78714-9347, USA.

Received: 1 July 2014 Accepted: 5 November 2014

Published: 19 November 2014 


\section{References}

1. Hansen JM: Oxidative stress as a mechanism of teratogenesis. Birth Defects Res C Embryo Today 2006, 78:293-307.

2. Kovacic $P$, Jacintho JD: Mechanisms of carcinogenesis: focus on oxidative stress and electron transfer. Curr Med Chem 2001, 8:773-796.

3. Huang B, Lei C, Wei C, Zeng G: Chlorinated volatile organic compounds (CI-VOCs) in the environment - sources, potential human health impacts, and current remediation technologies. Environ Int 2014, 71:118-138.

4. Elovaara E, Hemminki $K$, Vainio H: Effects of methylene chloride, trichloroethane, trichloroethylene, tetrachloroethylene and toluene on the development of chick embryos. Toxicology 1979, 12:111-119.

5. Loeber CP, Hendrix MJ, Diez De Pinos S, Goldberg SJ: Trichloroethylene: a cardiac teratogen in developing chick embryos. Pediatr Res 1988, 24:740-744.

6. Goldberg SJ, Dawson BV, Johnson PD, Hoyme HE, Ulreich JB: Cardiac teratogenicity of dichloroethylene in a chick model. Pediatr Res 1992, 32:23-26.

7. Johnson PD, Dawson BV, Goldberg SJ: Cardiac teratogenicity of trichloroethylene metabolites. J Am Coll Cardiol 1998, 32:540-545.

8. Johnson PD, Goldberg SJ, Mays MZ, Dawson BV: Threshold of trichloroethylene contamination in maternal drinking waters affecting fetal heart development in the rat. Environ Health Perspect 2003, 111:289-292.

9. Schwetz BA, Leong KJ, Gehring PJ: The effect of maternally inhaled trichloroethylene, perchloroethylene, methyl chloroform, and methylene chloride on embryonal and fetal development in mice and rats. Toxicol Appl Pharmacol 1975, 32:84-96.

10. Carney EW, Thorsrud BA, Dugard PH, Zablotny CL: Developmental toxicity studies in $\mathrm{Crl}: \mathrm{CD}$ (SD) rats following inhalation exposure to trichloroethylene and perchloroethylene. Birth Defects Res B Dev Reprod Toxicol 2006, 77:405-412

11. Chevrier C, Dananche B, Bahuau M, Nelva A, Herman C, Francannet C, Robert-Gnansia E, Cordier S: Occupational exposure to organic solvent mixtures during pregnancy and the risk of non-syndromic oral clefts. Occup Environ Med 2006, 63:617-623.

12. Desrosiers TA, Lawson CC, Meyer RE, Richardson DB, Daniels JL, Waters MA van Wijngaarden E, Langlois PH, Romitti PA, Correa A, Olshan A, and the National Birth Defects Prevention Study: Maternal occupational exposure to organic solvents during early pregnancy and risks of neural tube defects and orofacial clefts. Occup Environ Med 2012, 69:493-499.

13. Cordier S, Garlantezec R, Labat L, Rouget F, Monfort C, Bonvallot N, Roig B, Pulkkinen J, Chevrier C, Multigner L: Exposure during pregnancy to glycol ethers and chlorinated solvents and the risk of congenital malformations. Epidemiology 2012, 23:806-812.

14. Gilboa SM, Desrosiers TA, Lawson C, Lupo PJ, Riehle-Colarusso TJ, Stewart PA, van Wijngaarden E, Waters MA, Correa A, National Birth Defects Prevention Study: Association between maternal occupational exposure to organic solvents and congenital heart defects, National Birth Defects Prevention Study, 1997-2002. Occup Environ Med 2012, 69:628-635.

15. Yauck JS, Malloy ME, Blair K, Simpson PM, McCarver DG: Proximity of residence to trichloroethylene-emitting sites and increased risk of offspring congenital heart defects among older women. Birth Defects Res A Clin Mol Teratol 2004, 70:808-814.

16. Brender JD, Zhan FB, Suarez L, Langlois $\mathrm{PH}$, Moody K: Maternal residential proximity to waste sites and industrial facilities and oral clefts in offspring. J Occup Environ Med 2006, 48:565-572.

17. Langlois PH, Brender JD, Suarez L, Zhan FB, Mistry JH, Scheuerle A, Moody $\mathrm{K}$ : Maternal residential proximity to waste sites and industrial facilities and conotruncal heart defects in offspring. Paediatr Perinat Epidemiol 2009, 23:321-331.

18. Suarez L, Brender JD, Langlois PH, Zhan FB, Moody K: Maternal exposures to hazardous waste sites and industrial facilities and risk of neural tube defects in offspring. Ann Epidemiol 2007, 17:772-777.

19. Rasmussen SA, Olney RS, Holmes LB, Lin AE, Keppler-Noreuil KM, Moore CA, National Birth Defects Prevention Study: Guidelines for case classification for the National Birth Defects Prevention Study. Birth Defects Res A Clin Mol Teratol 2003, 67:193-201.

20. Zou B, Wilson JG, Zhan FB, Zeng Y: An emission-weighted proximity model for air pollution exposure assessment. Sci Total Environ 2009, 407:4939-4945.

21. Andersson T, Alfredsson L, Kallberg H, Zdravkovic S, Ahlbom A: Calculating measures of biological interactions. Eur J Epidemiol 2005, 20:575-579.
22. Laumon B, Martin JL, Collet P, Bertucat I, Verney MP, Robert E: Exposure to organic solvents during pregnancy and oral clefts: a case-control study. Reprod Toxicol 1996, 10:15-19.

23. Sonnenfeld N, Hertz-Picciotto I, Kaye WE: Tetrachloroethylene in drinking water and birth outcomes at the US Marine Corps Base at Camp Lejeune, North Carolina. Am J Epidemiol 2001, 154:902-908.

24. Canfield MA, Ramadhani TA, Langlois PH, Waller DK: Residential mobility patterns and exposure misclassification in epidemiologic studies of birth defects. J Expo Sci Environ Epidemiol 2006, 16:538-543.

25. Lupo PJ, Symanski E, Chan W, Mitchell LE, Waller DK, Canfield MA, Langlois $\mathrm{PH}$ : Differences in exposure assignment between conception and delivery: the impact of maternal mobility. Paediatr Perinat Epidemio/ 2010 24:200-208.

26. Brender JD, Zhan FB, Suarez L, Langlois P, Gilani Z, Delima I, Moody K: Linking environmental hazards and birth defects data. Int J Occup Environ Health 2006, 12:126-133.

27. Wilcox AJ: Fertility and Pregnancy. New York: Oxford University Press; 2010:99-114.

28. Lie RT, Wilcox AJ, Skjaerven R: A population-based study of the risk of recurrence of birth defects. N Engl J Med 1994, 331:1-4.

\section{doi:10.1186/1476-069X-13-96}

Cite this article as: Brender et al:: Maternal residential proximity to chlorinated solvent emissions and birth defects in offspring: a case-control study. Environmental Health 2014 13:96.

\section{Submit your next manuscript to BioMed Central and take full advantage of:}

- Convenient online submission

- Thorough peer review

- No space constraints or color figure charges

- Immediate publication on acceptance

- Inclusion in PubMed, CAS, Scopus and Google Scholar

- Research which is freely available for redistribution

Submit your manuscript at www.biomedcentral.com/submit
C Biomed Central 